\title{
On the Large Order Expansion for the Anharmonic Oscillators
}

\author{
V. R. Figerou ${ }^{\star}$ \\ Département de Physique Mathématique ${ }^{\star \star}$, U.S.T.L., F-34060 Montpellier Cedex, France
}

\begin{abstract}
Using functional approaches, we investigate the large- $K$ behaviour of the $K^{\text {th }}$ coefficient $E_{K}$ in the perturbation expansion for the ground-state energy $E(g)$ of the generalized anharmonic oscillator $X^{2 N}$ with internal $O(n)$-symmetry. We establish the equivalence between the pure functional approach and the method of Collins-Soper at any order in $\frac{1}{K}$. For that purpose, we first develop an algebraic treatment of perturbation series and prove a theorem on Borelsummable functions. Finally, we compute analytically the $1 / K$ and $1 / K^{2}$ corrections to the leading term for $N=2$.
\end{abstract}

\section{Introduction}

Some years ago, Bender and $\mathrm{Wu}[1]$ investigated the large order behaviour of the perturbation expansion of the energy levels of the anharmonic oscillator. More recently, in a series of works initiated by Lipatov [2], functional techniques have been applied to the determination of such behaviours. In the case of the generalized anharmonic oscillator, the quantity of main interest is the groundstate energy

$$
E(g)=-\lim _{T \rightarrow \infty} \frac{1}{T} \log \operatorname{Tr} \exp [-T H],
$$

where

$$
H=\frac{1}{2} \sum_{i=1}^{n} p_{i}^{2}+\frac{1}{2} \sum_{i=1}^{n} x_{i}^{2}+g\left(\sum_{i=1}^{n} x_{i}^{2}\right)^{N},
$$

and one looks for the large- $K$ behaviour of the coefficients $E_{K}$ in the asymptotic expansion

$$
E(g)=\sum_{K=0}^{\infty} E_{K} g^{K} .
$$

* Present address: Department of Physics, The Rockefeller University, New York, NY10021, USA

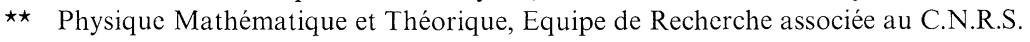


The functional approaches to this problem are based on a path integral representation of the partition function:

$$
Z(g, T)=\operatorname{Tr} \exp [-T H]=\int d[\mathbf{X}] \exp \left\{-\int_{-T / 2}^{T / 2} d t\left[\frac{1}{2}\left(\dot{\mathbf{X}}^{2}+\mathbf{X}^{2}\right)+g\left(\mathbf{X}^{2}\right)^{N}\right]\right\} .
$$

This integral has to be taken on the set of closed paths $\mathbf{X}(t)$ with period $T$

$$
\mathbf{X}(-T / 2)=\mathbf{X}(T / 2) \text {. }
$$

Among these approaches, we can schematically distinguish two methods. The first one was used by Collins and Soper [3] for $(N=2, n=1)$ and by Seznek [4] for $(N=2, n)$ with some variants. It combines the functional techniques with the dispersive approach used by Bender and $\mathrm{Wu}$ [1], which takes advantage of the known analyticity properties of the function $E(g)$ [5]. The other method is a pure functional approach which relies on the known Borel-summability properties of the function $E(g)$ [6]. It was used by Brézin et al. [7] to determine the leading term for any $N$ and $n$, as also by Auberson et al. [8] for $(N=2, n=1)$. These authors gave Feynman rules different from those of the first method. But they recovered the same contribution for the next-to-the-leading term.

The main purpose of this paper is to reconsider the problem for the Hamiltonian (1.2) and to prove the equivalence of both methods at any order in $1 / K$. In Sect. II, we apply the first one (Collins and Soper) just as it stands. In Sect. III, we apply and complete the second method (Auberson et al.) by using:

(a) A theorem on Borel-summable functions which is proved in Appendix A.

(b) An algebraic treatment of simplification of the perturbation series, which is performed in Sects. IV and V. This treatment consists in replacing simplification rules on series of Feynman diagrams by derivations on formal series.

Then, we will see that both methods are equivalent, for they lead indeed to the same final expression for $E_{K}$ at any order in $1 / K$. Finally, to test the validity of the functional approach, we give in Sect. VI the analytic results of the two first terms of the expansion for $(N=2, n)$ and of the third term for $(N=2, n=1)$. These results are in agreement with those of Bender and $\mathrm{Wu}$ [9] and Zinn-Justin [10], obtained by WKB or numerical methods.

\section{The First Method}

\section{A. Principle}

It is known [5] that $E(g)$ is analytic in the complex $g$ plane cut along the negative $g$ axis, and we assume that the partition function $Z(g, T)$ has the same analycity domain. Thus we can write a dispersion representation for both functions. The $K$-th order in $g$ is projected out by Cauchy's formula:

$$
\begin{aligned}
E_{K} & =-\frac{(-)^{K}}{2 i \pi} \int_{0}^{R} \frac{d \lambda}{\lambda^{K+1}} \operatorname{disc} E(-\lambda), \\
Z_{K}(T) & =-\frac{(-)^{K}}{2 i \pi} \int_{0}^{R} \frac{d \lambda}{\lambda^{K+1}} \operatorname{disc}(-\lambda, T) .
\end{aligned}
$$


Making use of Eq. (1.1), $\operatorname{disc} E(-\lambda)$ is related to $\operatorname{disc} Z(-\lambda, T)$ by

$$
\operatorname{disc} E(-\lambda)=-\lim _{T \rightarrow \infty} \frac{1}{T} \log \frac{1+1 / 2 \zeta^{-1} \operatorname{disc} Z}{1-1 / 2 \zeta^{-1} \operatorname{disc} Z},
$$

where $\zeta(-\lambda, T)$ and $\operatorname{disc} Z(-\lambda, T)$ are defined by

$$
Z(-\lambda \pm i \varepsilon, T) \equiv \zeta(-\lambda, T) \pm \frac{1}{2} \operatorname{disc} Z(-\lambda, T) .
$$

As $K \rightarrow \infty$, the factor $\lambda^{-(K+1)}$ becomes more and more singular at the endpoint $\lambda=0$ of the integrals (2.1) and (2.2). Thus if we know how $\operatorname{disc} Z(-\lambda, T)$ and $\zeta(-\lambda, T)$ behave for small $\lambda$, we can learn how $Z_{K}(-\lambda, T)$ and $E_{K}$ behave for large $K$. We use the saddle point method to evaluate $\operatorname{disc} Z(-\lambda, T)$ and $\zeta(-\lambda, T)$ for small $\lambda$.

B. Asymptotic Behaviour of $Z_{K}(T)$

(a) The action to minimize is for negative $g=-\lambda$

$$
S[\mathbf{X}]=\int_{-T / 2}^{T / 2} d t\left[\frac{1}{2}\left(\dot{\mathbf{X}}^{2}+\mathbf{X}^{2}\right)-\lambda\left(\mathbf{X}^{2}\right)^{N}\right] .
$$

The leading saddle points are easily found to be

$$
\mathbf{X}_{\mathrm{col}}(t-\tau, \mathbf{u})=\frac{1}{\sqrt{\mu}} x_{0}(t-\tau) \mathbf{u}
$$

where

(i)

(ii) $\tau$ is an arbitrary time origine,

$$
\mu=(2 N \lambda)^{\frac{1}{N-1}}
$$

(iii) $\mathbf{u}$ an arbitrary unit vector,

(iv) $x_{0}(t)$ the solution of the equation

$$
-\ddot{x}_{0}+x_{0}-x_{0}^{2 N-1}=0
$$

with negative energy and period $T$. We set:

$$
I_{0}=\int_{-T / 2}^{T / 2} x_{0}^{2 N}(t) d t, \quad k=\sqrt{\frac{2 N}{I_{0}}} .
$$

Then

$$
S\left[\mathbf{X}_{\mathrm{col}}\right]=\frac{N-1}{\mu k^{2}} .
$$

Around the saddle points, the quadratic terms are characterized by the second Frechet derivative $\mathscr{D}$ of the action.

$$
\begin{aligned}
\mathscr{D}_{i j} & =\mathscr{D}_{L} u_{i} u_{j}+\mathscr{D}_{T}\left(\delta_{i j}-u_{i} u_{j}\right), \\
\mathscr{D}_{L}\left(t_{1}, t_{2}\right) & =\left[-\frac{d^{2}}{d t_{1}^{2}}+1-(2 N-1) x_{0}^{2 N-2}\left(t_{1}\right)\right] \delta\left(t_{1}-t_{2}\right), \\
\mathscr{D}_{T}\left(t_{1}, t_{2}\right) & =\left[-\frac{d^{2}}{d t_{1}^{2}}+1-x_{0}^{2 N-2}\left(t_{1}\right)\right] \delta\left(t_{1}-t_{2}\right) .
\end{aligned}
$$


(b) Since the action is invariant under "time translations" and "space rotations", $\dot{x}_{0}$ and $x_{0}$ are respectively eigenvectors with eigenvalue 0 of the operators $\mathscr{D}_{L}$ and $\mathscr{D}_{T}$. These are the so-called zero modes. To take care of them when performing the integration, one can apply collective coordinates, Faddeev-Popov or other standard methods [11]. We replace the variables $\mathbf{X}(t)$ by $\tau,(n-1)$ angles $\theta_{i}$ which characterize the direction $\mathbf{u}$ and $\mathbf{Y}(t)$ orthogonal to the zero modes at the point $\mathbf{X}_{\mathrm{col}}(t-\tau, \mathbf{u})$

$$
\begin{gathered}
\mathbf{X}(t)=\mathbf{X}_{\mathrm{col}}(t-\tau, \mathbf{u})+\mathbf{Y}(t-\tau, \mathbf{u}), \\
\left\langle\frac{\partial}{\partial \tau} \mathbf{X}_{\mathrm{col}} \mid \mathbf{Y}\right\rangle=0, \\
\left\langle\frac{\partial}{\partial \theta_{i}} \mathbf{X}_{\mathrm{col}} \mid \mathbf{Y}\right\rangle=0 \quad(i=1, \ldots, n-1) .
\end{gathered}
$$

[The scalar product $\langle\mathbf{f} \mid \mathbf{g}\rangle$ of two paths $\mathbf{f}$ and $\mathbf{g}$ is defined by $\langle\mathbf{f} \mid \mathbf{g}\rangle=\int_{-T / 2}^{T / 2} \mathbf{f}(t) \cdot \mathbf{g}(t) d t$.] Since $\left\langle\mathbf{X}_{\mathrm{col}} \mid \mathbf{X}_{\mathrm{col}}\right\rangle$ is independent of $\tau$, $\mathbf{u}$, Eqs. (2.10) imply for any path $\mathbf{X}(t)$ :

$$
\begin{gathered}
\mathbf{u} \cdot \int_{-T / 2}^{T / 2} d t \dot{x}_{0}(t-\tau) \mathbf{X}(t) d t=0, \\
\frac{\partial \mathbf{u}}{\partial \theta_{i}} \cdot \int_{-T / 2}^{T / 2} d t x_{0}(t-\tau) \mathbf{X}(t) d t=0 .
\end{gathered}
$$

Generally, $\left\langle\mathbf{X}_{\tau}\right\rangle=\int_{-T / 2}^{T / 2} d t x_{0}(t-\tau) \mathbf{X}(t)$ will not vanish for any $\tau$ and Eq. (2.11b) have two solutions

$$
\mathbf{u}= \pm \frac{\left\langle\mathbf{X}_{\tau}\right\rangle}{\left\|\left\langle\mathbf{X}_{\tau}\right\rangle\right\|}
$$

Equation (2.11a) can then be written

$$
\frac{d}{d \tau}\left\|\left\langle\mathbf{X}_{\tau}\right\rangle\right\|=0
$$

$\left\|\left\langle\mathbf{X}_{\tau}\right\rangle\right\|$ is a periodic function with period $T$. Its derivative has at least two zeros on the period $T$. Since $\mathbf{u}$ is defined up to a sign, for any path $\mathbf{X}(t)$, there are generally at least four values of parameters $(\tau, \mathbf{u})$ to make the decomposition (2.9).

The integration domain for $\mathbf{Y}(t)$ is perhaps not independent of $(\tau, \mathbf{u})$. But since only the paths $\mathbf{X}(t)$ not too far from one of the saddle points contribute significantly, we can extend the integration of $\mathbf{Y}(t)$ over the whole hyperplan orthogonal to the zero modes. The Jacobian of the transformation (2.9) is found to be

$$
J=\sum_{i=1}^{n-2}\left(\sin \theta_{i}\right)^{n-1-i} \frac{\left\langle\mathbf{X}_{\mathrm{col}} \mid \mathbf{X}\right\rangle^{n-2}}{\left\|\mathbf{X}_{\mathrm{col}}\right\|^{n-1}\left\|\dot{\mathbf{X}}_{\mathrm{col}}\right\|}\left[\left\langle\mathbf{X}_{\mathrm{col}} \mid \mathbf{X}\right\rangle\left\langle\dot{\mathbf{X}}_{\mathrm{col}} \mid \dot{\mathbf{X}}\right\rangle-\langle\mathbf{X}|M| \mathbf{X}\rangle\right]
$$

where $M$ is the linear operator

$$
M_{i j}\left(t_{1}, t_{2}\right)=\left(\dot{\mathbf{X}}_{\mathrm{col}}\left(t_{1}\right) \cdot \dot{\mathbf{X}}_{\mathrm{col}}\left(t_{2}\right)\right)\left(\delta_{i j}-u_{i} u_{j}\right)
$$


$J$ does not depend on $(\tau, \mathbf{u})$. The integrations with respect to $\tau$ and over the directions of $\mathbf{u}$ give respectively the factors $T$ and $\frac{2 \pi^{n / 2}}{\Gamma(n / 2)}$. The integration over the orthogonal hyperplan gives the factor $\left(\frac{-i}{\sqrt{-2 \pi \Delta_{L}}}\right)\left(\frac{1}{\sqrt{2 \pi \Delta_{T}}}\right)^{n-1}$ where $\Delta_{L}$ and $\Delta_{T}$ are the determinants of $\mathscr{D}_{L}$ and $\mathscr{D}_{T}$ in the subspaces orthogonal to their respective zero modes. Then we obtain for the leading term of $\operatorname{disc} Z(-\lambda, T)$

$$
\begin{aligned}
\operatorname{disc} Z(-\lambda, T) \sim & \frac{-i T}{\sqrt{-2 \pi \Delta_{L}}} \frac{2 \pi^{n / 2}}{\Gamma(n / 2)}\left(\frac{1}{\sqrt{2 \pi \Delta_{T}}}\right)^{n-1}\left\|\dot{x}_{0}\right\|\left\|x_{0}\right\|^{n-1} \mu^{-n / 2} \\
& \cdot \exp \left(-\frac{N-1}{\mu k^{2}}\right)[1+O(\mu)] .
\end{aligned}
$$

(c) The next-to-the-leading terms can be evaluated by standard Feynman diagram techniques. The propagator $G\left(t, t^{\prime}\right)$ is the inverse of the linear operator $\mathscr{D}$ in the subspace orthogonal to the zero modes. As for the vertices, they come from the expansion in powers of $\mathbf{Y}=\mathbf{X}-\mathbf{X}_{\mathrm{col}}$ of the action and of the Jacobian. So, we define $(2 N+n)$ types of vertices:

$$
\begin{aligned}
I_{r}= & \frac{(2 N-r) !}{(2 N) !} \int_{-T / 2}^{T / 2} d t \frac{\partial}{\partial u_{m_{1}}} \ldots \frac{\partial}{\partial u_{m_{r}}}\left(\sum_{i=1}^{n} u_{i}^{2}\right)_{\|\mathbf{u}\|=1}^{N} \\
& \cdot x_{0}^{2 N-r}(t) Y_{m_{1}}(t) \ldots Y_{m_{r}}(t) \quad(r=3,4,2 N), \\
U= & \int \frac{x_{0}(t) \mathbf{u} \cdot \mathbf{Y}(t)}{\left\|x_{0}\right\|^{2}} d t, \\
V= & \int \frac{-\ddot{x}_{0}(t) \mathbf{u} \cdot \mathbf{Y}(t)}{\left\|\dot{x}_{0}\right\|^{2}} d t \\
W_{i}= & \int \frac{\dot{x}_{0}(t) \mathbf{i} \cdot \mathbf{Y}(t)}{\left\|\dot{x}_{0}\right\|\left\|x_{0}\right\|} d t \quad(i=1, \ldots, n),
\end{aligned}
$$

where $\mathbf{i}$ is the unit vector

$$
\left(\begin{array}{c}
i \\
\downarrow \\
\mathbf{i}=0,0, \ldots, 1, \ldots, 0
\end{array}\right) .
$$

The propagator $G\left(t, t^{\prime}\right)$ and the $(2 N+n)$ vertices completely define our Feynman rules.

(d) By substituting the expression of $\operatorname{disc} Z(-\lambda, T)$ in Eq. (2.2), we obtain for the asymptotic behaviour of $Z_{K}(T)$ after integration

$$
\begin{aligned}
Z_{K}(T)= & \frac{1}{2 \pi}\left(-k^{2 N} I_{0}\right)^{K} \frac{T}{\sqrt{-2 \pi \Delta_{L}}} \frac{2 \pi^{n / 2}}{\Gamma(n / 2)}\left(\frac{1}{\sqrt{2 \pi \Delta_{T}}}\right)^{n-1} \\
& \cdot \frac{\left\|\dot{x}_{0}\right\|\left\|x_{0}\right\|^{n-1} k^{n}}{(N-1)^{(N-1) K+n / 2-1}} \sum_{\ell=0}^{\infty} \Gamma\left[(N-1) K+\frac{n}{2}-\ell\right]\left(\frac{N-1}{k^{2}}\right)^{\ell} b_{t}(T),
\end{aligned}
$$


where $B(\mu, T)=\sum_{\ell=0}^{\infty} b_{\ell}(T) \mu^{\ell}$ is the sum over the Feynman diagrams built with the propagator $G\left(t, t^{\prime}\right)$ and generated by the expansion in $\mu$ of the expression

$$
\begin{aligned}
B(\mu, T)= & \exp \left(\sum_{r=3}^{2 N} \frac{C_{2 N}^{r}}{2 N} \mu^{\frac{r-2}{2}} I_{r}\right)(1+\sqrt{\mu} U)^{n-2} \\
& \cdot\left[(1+\sqrt{\mu} U)(1+\sqrt{\mu} V)-\mu\left(\delta_{i j}-u_{i} u_{j}\right) W_{i} W_{j}\right] .
\end{aligned}
$$

(Here and in the following, $C_{a}^{b}$ denotes the binomial coefficient $\frac{a !}{b !(a-b) !}$ )

C. Asymptotic Behaviour of $E_{K}$

For $\zeta(-\lambda, T)$, the saddle point is the path $\mathbf{X}(t) \equiv 0$. The expansion of $\zeta(-\lambda, T)$ for small $\lambda$ is just the ordinary perturbation expansion of $Z(g)$ with $-\lambda$ substituted for $g$ term by term

$$
\zeta(-\lambda, T) \sim\left(\frac{1}{2 \sinh T / 2}\right)^{n} \exp \sum_{\ell=1}^{\infty} \frac{\lambda^{\ell}}{\ell !} \tilde{\mathscr{R}}(\ell),
$$

where $\tilde{\mathscr{R}}(\ell)$ represents the sum over connected Feynman diagrams constructed with $\ell 2 N$ leg-Vertices and "free" propagators $\left(G_{0}\right)_{i j}=\left(-\frac{d^{2}}{d t^{2}}+1\right)^{-1} \delta_{i j}$.

Making the change of variables $(2.5 b)$, we obtain for the product $\zeta^{-1} \operatorname{disc} Z$ :

$$
\zeta^{-1} \operatorname{disc} Z \sim-2 i T \frac{C(T)}{k^{n}} \mu^{-n / 2} \exp \left(-\frac{N-1}{\mu k^{2}}\right) A(\mu, T)
$$

with

$$
C(T)=\frac{\pi^{n / 2}}{\Gamma(n / 2)} \frac{2 \sinh T / 2}{\sqrt{-2 \pi \Delta_{L}}}\left(\frac{2 \sinh T / 2}{\sqrt{2 \pi \Delta_{T}}}\right)^{n-1}\left\|\dot{x}_{0}\right\|\left\|x_{0}\right\|^{n-1} k^{n}
$$

and

$$
A(\mu, T)=\sum_{\ell=0}^{\infty} a_{\ell}(T) \mu^{\ell}=B(\mu, T) \exp -\sum_{\ell=1}^{\infty}\left(\frac{\mu^{N-1}}{2 N}\right)^{\ell} \frac{\tilde{\mathscr{R}}(\ell)}{\ell !} .
$$

In the limit $T \rightarrow \infty, x_{0}(t)$ satisfies

$$
x_{0}^{2 N-2}(t)=\frac{N}{\cosh ^{2}(N-1) t},
$$

$I_{0}$ and $C(T)$ approach limiting values which have been evaluated by Brézin et al. [7]

$$
\begin{gathered}
I_{0} \rightarrow \frac{N^{\frac{N}{N-1}} 2^{\frac{N+1}{N-1}}}{N-1} \frac{\Gamma^{2}(N / N-1)}{\Gamma(2 N / N-1)}, \\
C(T) \rightarrow \frac{(N-1)^{n / 2}}{\Gamma(n / 2)}\left[\frac{\Gamma(2 N / N-1)}{\Gamma^{2}(N / N-1)}\right]^{n / 2},
\end{gathered}
$$

and we will see in the next section that the coefficients $a_{\ell}(T)$ tend to finite limits $a_{\ell}$. 
If we assume the commutativity of the limits $\lambda \rightarrow 0$ and $T \rightarrow \infty$, we can deduce from (2.3) the asymptotic expansion of $\operatorname{disc} E$ for small $\lambda$, and by integration, the asymptotic expansion of $E_{K}$ at large order. Finally, one finds

$$
\begin{aligned}
E_{K}= & -\frac{1}{\pi}\left(-\frac{1}{2}\right)^{K} \frac{N-1}{\Gamma(n / 2)}\left[\frac{\Gamma(2 N / N-1)}{\Gamma^{2}(N / N-1)}\right]^{(N-1) K+n / 2} \\
& \cdot \sum_{\ell=0}^{\infty} \Gamma\left(K(N-1)+\frac{n}{2}-\ell\right) D^{\ell} a_{\ell}
\end{aligned}
$$

with

$$
D=\lim _{T \rightarrow \infty} \frac{N-1}{k^{2}}=(4 N)^{\frac{1}{N-1}} \frac{\Gamma^{2}(N / N-1)}{\Gamma(2 N / N-1)}
$$

and

$$
A(\mu)=\sum_{\ell=0}^{\infty} a_{\ell} \mu^{\ell}=\lim _{T \rightarrow \infty} A(\mu, T) .
$$

Equations (2.25) generalize results previously obtained by Bender and Wu [9]. It now remains to prove the existence of the limit $A(\mu)$.

D. Finite Limit of $A(\mu, T)$

Let us expand the series $B(\mu, T)$

$$
\begin{aligned}
B(\mu, T)= & \sum_{\alpha, \beta_{3}, \ldots, \beta_{2 N-1}} \frac{1}{\alpha !}\left(\frac{\mu^{N-1}}{2 N} I_{2 N}\right)^{\alpha} \prod_{r=3}^{2 N-1} \frac{1}{\beta_{r} !}\left(\frac{C_{2 N}^{r}}{2 N} \mu^{\frac{r-2}{2}} I_{r}\right)^{\beta_{r}} \\
& \cdot(1+U \sqrt{\mu})^{n-2}\left[(1+U \sqrt{\mu})(1+V \sqrt{\mu})-\mu\left(\delta_{i j}-u_{i} u_{j}\right) W_{i} W_{j}\right] .
\end{aligned}
$$

We denote by $\mathscr{R}(\ell)$ the sum over connected Feynman diagrams built with $\ell$ vertices $I_{2 N}$ and the propagator $G$. To express the monomial

$$
I_{2 N}^{\alpha} \prod_{r=3}^{2 N-1} I_{r}^{\beta_{r}} U^{\gamma} V^{\delta}\left[\left(\delta_{i j}-u_{i} u_{j}\right) W_{i} W_{j}\right]^{\varepsilon}
$$

in terms of the components $\mathscr{R}(\ell)$ is a simple combinatorial problem. We have to distribute the $\alpha$ vertices $I_{2 N}$ amongst

$\alpha_{1}$ sets of the connected diagrams built with exactly 1 vertex $I_{2 N}$

$\alpha_{2}$ sets of the connected diagrams built with exactly 2 vertices $I_{2 N}$

$\alpha_{\ell}$ sets of the connected diagrams built with exactly $\ell$ vertices $I_{2 N}$

and $\alpha_{0}$ remaining vertices $I_{2 N}$.

Each monomial (2.27) can be decomposed into

$$
\sum_{\alpha_{0}+\sum_{\ell} \ell \alpha_{\ell}=\alpha} \frac{\alpha !}{\alpha_{0} ! \alpha_{1} ! \ldots \alpha_{\ell} ! \ldots} \prod_{\ell \geqq 1}\left[\frac{\mathscr{R}(\ell)}{\ell !}\right]^{\alpha_{\ell}} \mathscr{F},
$$


where $\mathscr{F}$ is a function of the connected components containing at least one of the vertices $I_{r}(3 \leqq r \leqq 2 N-1), U, V$ or $\left[\left(\delta_{i j}-u_{i} u_{j}\right) W_{i} W_{j}\right]$. Then $\mathscr{F}$ depends only on the indices $\alpha_{0}, \beta_{r}, \gamma, \delta, \varepsilon$, but not on $\alpha_{\ell}(\ell \geqq 1)$. Therefore, substituting into Eq. (2.26) the expression (2.28) for each monomial, we can factorize the components $\mathscr{R}(\ell)$ and finally we find

$$
B(\mu, T)=\mathscr{F}^{\prime}(\mu) \exp \left[\sum_{\ell=1}^{\infty}\left(\frac{\mu^{N-1}}{2 \mathrm{~N}}\right)^{\ell} \frac{\mathscr{R}(\ell)}{\ell !}\right]
$$

with $\mathscr{F}^{\prime}(\mu)$ characterized as $\mathscr{F}$. Inserting (2.29) into (2.22), we have

$$
A(\mu, T)=\mathscr{F}^{\prime}(\mu) \exp \left[\sum_{\ell=1}^{\infty}\left(\frac{\mu^{N-1}}{2 \mathrm{~N}}\right) \frac{\mathscr{R}(\ell)-\tilde{\mathscr{R}}(\ell)}{\ell !}\right] .
$$

By proceeding exactly as Seznek [4] did for $(N=2, n)$, we can show that, at the $T \rightarrow \infty$ limit, the only connected divergent graphs built with the propagator $G$ are those containing only $2 N$-leg vertices. These divergences are not worse than linear. Moreover, such connected graphs diverge exactly as do the same graphs built with the free propagator $G_{0}$, so that their difference is finite. This last property comes from the fact that $G\left(t, t^{\prime}\right)$ and $G_{0}\left(t, t^{\prime}\right)$ have the same behaviour for large $t$ and $t^{\prime}$. The existence of the limit $T \rightarrow \infty$ for $A(\mu, T)$ easily follows.

\section{The Second Method}

\section{A. Expansion About Saddle Points}

Expanding $Z(g, T)$ in powers of the coupling constant $g$

$$
Z(g, T)=\sum_{K=0}^{\infty} Z_{K}(T) g^{K},
$$

one gets

$$
Z_{K}(T)=\frac{(-)^{K}}{K !} \int d[\mathbf{X}] \exp -S_{K}[\mathbf{X}]
$$

where

$$
S_{K}[\mathbf{X}]=\frac{1}{2} \int_{-T / 2}^{T / 2} d t\left(\dot{\mathbf{X}}^{2}+\mathbf{X}^{2}\right)-K \log \int_{-T / 2}^{T / 2} d t\left(\mathbf{X}^{2}\right)^{N} .
$$

The leading contribution to the action in the large- $T$ limit comes from the trajectories

$$
\mathbf{X}_{\mathrm{col}}(t-\tau, \mathbf{u})=k \sqrt{K} x_{0}(t-\tau) \mathbf{u},
$$

where $x_{0}, \mathbf{u}, \tau, I_{0}$, and $k$ are defined as in the first method. Then

$$
S_{K}\left[\mathbf{X}_{\mathrm{col}}\right]=-K \log \left(k^{2 N} I_{0}\right)-N K \log (K / e) .
$$

If we expand the functional integral for $Z_{K}(T)$ about the saddle points, we obtain

$$
\begin{aligned}
Z_{K}(T)= & \frac{(-)^{K}}{K !}\left(k^{2 N} I_{0}\right)^{K}\left(\frac{K}{e}\right)^{N K} \int d[\mathbf{Y}] \exp \left[-\frac{1}{2}\langle\mathbf{Y}|(\mathscr{D}+|\mathbf{P}\rangle\langle\mathbf{P}|)| \mathbf{Y}\rangle\right] \\
& \cdot \exp \left[K \log \left(\sum_{r=0}^{2 N} \frac{C_{2 N}^{r}}{k^{r} K^{r / 2} I_{0}} I_{r}\right)-\frac{2 N \sqrt{K}}{k I_{0}} I_{1}-\frac{C_{2 N}^{2}}{k^{2} I_{0}} I_{2}+\frac{2 N^{2}}{k^{2} I_{0}^{2}} I_{1}^{2}\right]
\end{aligned}
$$


where

(i) $\mathscr{D}$ is the linear operator $(2.8)$,

(ii) $\mathbf{P}=k x_{0}^{2 N-1} \mathbf{u}$,

(iii) $I_{r}$ is defined by $(2.16 \mathrm{a})$ with $(1 \leqq r \leqq 2 N)$.

The operator $\mathscr{D}+|\mathbf{P}\rangle\langle\mathbf{P}|$ can be decomposed into a longitudinal and a transverse part

$$
(\mathscr{D}+|\mathbf{P}\rangle\langle\mathbf{P}|)_{i j}=\overline{\mathscr{D}}_{L} u_{i} u_{j}+\overline{\mathscr{D}}_{T}\left(\delta_{i j}-u_{i} u_{j}\right) .
$$

The functions $\dot{x}_{0}$ and $x_{0}$ are respectively eigenvectors with eigenvalue 0 of $\overline{\mathscr{D}_{L}}$ and $\overline{\mathscr{D}_{T}}$. To extract the zero modes, we proceed exactly as in Sect. II. Therefore the integration over the quadratic term gives the same Jacobian (2.14) and the factor

$$
T \frac{2 \pi^{n / 2}}{\Gamma(n / 2)} \frac{1}{\sqrt{2 \pi \bar{\Delta}_{L}}}\left(\frac{1}{\sqrt{2 \pi \Delta_{T}}}\right)^{n-1},
$$

where $\bar{\Delta}_{L}$ is the determinant of $\overline{\mathscr{D}}_{L}$ in the subspace orthogonal to $\dot{x}_{0}$. Since $x_{0}^{2 N-1}$ belongs to this subspace, we can write

$$
\overline{\Delta_{L}}=\Delta_{L}\left(1+\left\langle\mathbf{P}\left|\mathscr{D}_{L}^{-1}\right| \mathbf{P}\right\rangle\right)
$$

Noticing that

$$
\mathscr{D}_{L} x_{0}(t)=-2(N-1) x_{0}^{2 N-1}(t)
$$

we obtain

$$
\bar{\Delta}_{L}=-\frac{\Delta_{L}}{N-1}
$$

The next-to-the leading terms can be evaluated by standard diagram techniques again. The propagator $\mathscr{G}\left(t, t^{\prime}\right)$ is the inverse of the linear operator $\mathscr{D}+|\mathbf{P}\rangle\langle\mathbf{P}|$ in the subspace orthogonal to the zero modes. It is given by

$$
\mathscr{G}=\mathrm{G}-\frac{\mathrm{G}|\mathbf{P}\rangle\langle\mathbf{P}| G}{1+\langle\mathbf{P}|G| \mathbf{P}\rangle},
$$

where $G$ is the propagator of the first method. As for the vertices, they come from the expansion in powers of $\mathbf{Y}=\mathbf{X}-\mathbf{X}_{\mathrm{col}}$ of the action and of the Jacobian. So we have $(2 N+n+2)$ types of vertex $I_{r}(1 \leqq r \leqq 2 N), U, V$, and $W_{i}(1 \leqq i \leqq n)$ defined by (2.16).

We are now in a position to write down the asymptotic behaviour of $Z_{K}(T)$

$$
\begin{aligned}
Z_{K}(T) \sim & \frac{\left(-k^{2 N} I_{0}\right)^{K}}{K !}\left(\frac{K}{e}\right)^{N K} K^{n / 2} \frac{T \sqrt{N-1}}{\sqrt{-2 \pi \Delta_{L}}} \frac{2 \pi^{n / 2}}{\Gamma(n / 2)} \\
& \cdot\left(\frac{1}{\sqrt{2 \pi \Delta_{T}}}\right)^{n-1}\left\|\dot{x}_{0}\right\|\left\|x_{0}\right\|^{n-1} k^{n} S_{I},
\end{aligned}
$$

where $S_{I}$ is the sum over Feynman diagrams built with the propagator $\mathscr{G}\left(t, t^{\prime}\right)$ and 
generated by the expansion in $\frac{1}{\sqrt{K}}$ of the expression

$$
\begin{aligned}
S_{I}= & \exp \left[K \log \left(\sum_{r=0}^{2 N} \frac{C_{2 N}^{r}}{k^{r} K^{r / 2} I_{0}} I_{r}\right)-\frac{2 N \sqrt{K}}{k I_{0}} I_{1}-\frac{C_{2 N}^{2}}{k^{2} I_{0}} I_{2}\right. \\
& \left.+\frac{2 N^{2}}{k^{2} I_{0}^{2}} I_{1}^{2}\right] J\left(U, V, \ldots, W_{i} \ldots\right)
\end{aligned}
$$

with

$$
J\left(U, V, \ldots, W_{i} \ldots\right)=\left(1+\frac{1}{k \sqrt{K}} U\right)^{n-2}\left[\left(1+\frac{1}{k \sqrt{K}} U\right)\left(1+\frac{1}{k \sqrt{K}} V\right)-\frac{\delta_{i j}-u_{i} u_{j}}{k^{2} K} W_{i} W_{j}\right] .
$$

\section{B. Simplification Rules}

From Eqs. (3.7), (3.10), (3.12), it follows that

$$
\begin{aligned}
& G\left|x_{0}^{2 N-1} \mathbf{u}\right\rangle=-\frac{1}{2(N-1)}\left|x_{0} \mathbf{u}\right\rangle, \\
& \mathscr{G}=G+\frac{N}{2(N-1) I_{0}}\left|x_{0} \mathbf{u}\right\rangle\left\langle x_{0} \mathbf{u}\right| .
\end{aligned}
$$

The derivative $\frac{\partial}{\partial u_{m_{2}}} \ldots \frac{\partial}{\partial u_{m_{r}}}\left(\sum_{1}^{n} u_{i}^{2}\right)^{N}$ is a homogeneous function of degree $(2 N-r+1)$. Therefore, by substituting $x_{0}(t) u_{m_{1}}$ for $Y_{m_{1}}(t)$ into $(2.16 \mathrm{a})$ and by applying Euler's formula, one gets

$$
\begin{gathered}
\frac{(2 N-r) !}{(2 N) !} \int_{-T / 2}^{T / 2} d t \sum_{m_{1} \ldots m_{r}} \frac{\partial}{\partial u_{m_{1}}} \frac{\partial}{\partial u_{m_{2}}} \ldots \frac{\partial}{\partial u_{m_{r}}}\left(\sum_{1}^{n} u_{i}^{2}\right)_{\|\mathbf{u}\|=1}^{N} \\
\cdot x_{0}^{2 N-r}(t) x_{0}(t) u_{m_{1}} Y_{m_{2}}(t) \ldots Y_{m_{r}}(t)=I_{r-1} .
\end{gathered}
$$

We will represent the propagators as in Fig. 1

Fig. 1

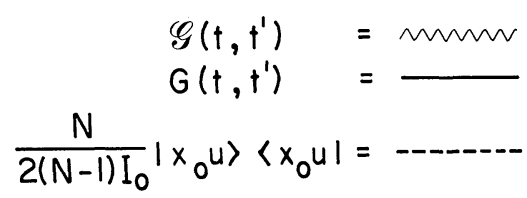

According to (3.16) for any line in a diagram, we can proceed to the decomposition :

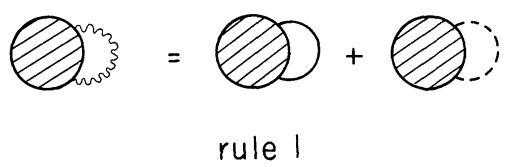

We obtain a new sum of Feynman diagrams where there are only lines and -----. Equations (3.15), (3.17), together with the particular forms of the propagator - - and the vertices $I_{r}, U, V, W_{i}$ imply on these diagrams the 
following simplification rules:
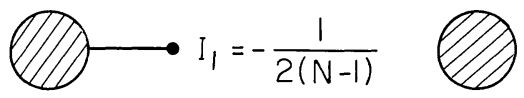

rule 2

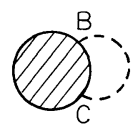

$=\frac{N}{2(N-1) I_{0}}$

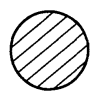

rule 3

where

(i) $B, C$ may represent the same vertex (loop),

(ii) after simplification, the vertices without leg are scalars given by

$$
\text { - }=I_{0}, \quad U_{0}=V_{0}=1, \quad\left(W_{i}\right)_{0}=0, \quad i=1, \ldots, n .
$$

For example we have

$$
\begin{aligned}
& U \longrightarrow I_{1}=\frac{-1}{2(N-1)} U_{0}=\frac{-1}{2(N-1)}, \\
& V-I_{1}=\frac{N}{2(N-1) I_{0}} V_{0} I_{0}=\frac{N}{2(N-1)} .
\end{aligned}
$$

We observe that the application of the simplification rules eliminates the lines and the vertices $I_{1}$. In Sect. V, we will show the following important result: the final expression of the series of Feynman diagrams $S_{I}$ after simplification is

$$
S_{I}=\frac{1}{2 \pi} \frac{K !}{K^{n / 2}}\left(\frac{e}{K}\right)^{N K} \sum_{\ell=0}^{\infty} \frac{\Gamma\left[K(N-1)+\frac{n}{2}-\ell\right]}{(N-1)^{(N-1) K+n / 2-1 / 2}}\left(\frac{N-1}{k^{2}}\right)^{\ell} b_{\ell}(T),
$$

where, as in Sect. II, $B(\mu, T)=\sum_{\ell=0}^{\infty} b_{\ell}(T) \mu^{\ell}$ is the sum over Feynman diagrams built with the propagator $G\left(t, t^{\prime}\right)$ and generated by the expansion in $\mu$ of the expression (2.18).

Substituting (3.18) for $S_{I}$ into (3.13), we obtain for the asymptotic behaviour of $Z_{K}(T)$ at large order and at fixed $T$ the expansion (2.17) of Sect. II. Thus, we obtain exactly the result that was established by the previous method.

\section{Asymptotic Behaviour of $E_{K}$}

We have now to calculate the asymptotic behaviour of $E_{K}$ at large order. It is known [6] that $\mathscr{E}(h)=E\left(h^{N-1}\right)$ is Borel-summable in the variable $h=g^{\frac{1}{N-1}}$, and we assume that the same is true for $\mathscr{Z}(h, T)=Z\left(h^{N-1}, T\right)$. Then, according to a theorem proved by Auberson et al. [12], $\log \mathscr{Z}(h, T)$ is also Borel-summable. On the other hand, $\mathscr{Z}(h, T)$ satisfies the conditions of the theorem of Appendix A. 
Then, by applying this theorem, we have:

with, at the order $\left(\frac{1}{K}\right)^{r}, c_{K}(T)$ given by

$$
\log Z(g, T)=\sum_{K=0}^{\infty} c_{K}(T) g^{K}
$$

$$
c_{K}(T)=\sum_{i=0}^{r / N-1} Z_{K-i} d_{i}
$$

where $d_{i}$ is the general term of the series

$$
\sum_{i=0}^{\infty} d_{i} g^{i}=\left.\frac{d}{d u} \log u\right|_{u=\sum_{0}^{\infty} Z_{i} g^{2}}=\left[\sum_{0}^{\infty} Z_{i} g^{i}\right]^{-1} .
$$

But $Z_{K}(T)$ is itself given by (2.17). Inserting (2.17) into (3.19b), we get

$$
c_{K}(T) \sim \frac{T C(T)\left(-k^{2 N} I_{0}\right)^{K}}{\pi} \sum_{\ell=0}^{r} \frac{\Gamma\left[K(N-1)+\frac{n}{2}-\ell\right]}{(N-1)^{(N-1) K+n / 2-1}}\left(\frac{N-1}{k^{2}}\right)^{\ell} a_{\ell}(T)
$$

with

$$
a_{\ell}(T)=Z_{0}(T) \sum_{i(N-1)+j=\ell}\left(-\frac{1}{2 N}\right)^{i} d_{i} b_{j}(T) ;
$$

$a_{\ell}(T)$ is the general term of the product of two series

$$
A(\mu, T)=\sum_{0}^{\infty} a_{\ell}(T) \mu^{\ell}=Z_{0}(T) B(\mu, T) \sum_{\ell=0}^{\infty}\left(-\frac{\mu^{N-1}}{2 N}\right)^{\ell} d_{\ell} .
$$

We can represent $Z(g, T)$ by the ordinary perturbation expansion

$$
Z(g, T)=Z_{0}(T) \exp \sum_{\ell=1}^{\infty} \frac{(-g)^{\ell} \tilde{\mathscr{R}}(\ell)}{\ell !},
$$

where $\tilde{\mathscr{R}}(\ell)$ represents the sum over connected Feynman diagrams constructed with $\ell 2 N$-leg vertices and the "free" propagators $G_{0}$. Combining Eqs. (3.19c) and (3.22), we find

$$
\sum_{\ell=0}^{\infty} d_{\ell} g^{\ell}=\frac{1}{Z_{0}(T)} \exp \left(-\sum_{\ell=1}^{\infty}(-\mathrm{g})^{\ell} \frac{\tilde{\mathscr{R}}(\ell)}{\ell !}\right)
$$

and, from Eqs. (3.21) and (3.23), we recover the expression (2.22) for $A(\mu, T)$.

In the limit $T \rightarrow \infty$, as in Sect. II, $x_{0}(t)$ satisfies Eq. (2.23), $I_{0}$ and $C(T)$ approach the limiting values (2.24), and the coefficients $a_{\ell}(T)$ remain finite. If we assume the commutativity of the limits $K \rightarrow \infty$ and $T \rightarrow \infty$, we can deduce the asymptotic behaviour of $E_{K}$

$$
E_{K}=-\lim _{T \rightarrow \infty} \frac{c_{K}(T)}{T} .
$$

By inserting (3.20) into (3.24) and by taking the $T \rightarrow \infty$ limit, we recover for $E_{K}$ the asymptotic behaviour (2.25) that was established by the previous method. Therefore, the equivalence of the two methods is proved. 


\section{Study of Simplifications}

\section{A. Preliminary}

In this section, we will be concerned with the study of Feynman diagram series where Feynman rules are defined by

(i) A symmetric propagator $\mathscr{G}$ satisfying Eqs. (3.15) and (3.16).

(ii) Vertices $I_{i}(1 \leqq i \leqq 2 N)$ defined by $(2.16 \mathrm{a})$.

(iii) An arbitrary number $n^{\prime}$ of completely symmetric tensors $\stackrel{(j)}{T}(t)$ of rank $r_{j}$ $\left(j=1, \ldots, n^{\prime}\right)$, and their associated families of vertices $\stackrel{(j)}{U}_{i}$, with $i$ legs $\left(0 \leqq i \leqq r_{j}\right)$ defined by

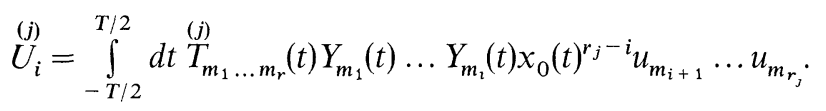

(In fact $\stackrel{(j)}{U_{0}}$ doesn’t define a vertex and is just a scalar.)

In the following, we will generally omit the family index $(j)$. The series $S_{I}(3.14)$ involves $(n+2)$ families of rank $r=1$, namely $U, V, \ldots, W_{l} \ldots$.

It is easy to see that Eqs. (3.15), (3.16), together with the particular form of the vertices $I_{i}, U_{i}$ imply the validity of the rules $(1,2,3)$ on these Feynman diagrams. In the two last ones, a vertex behaves as follows

$$
\begin{aligned}
I_{i} & \rightarrow I_{i-1} \\
U_{i} & \rightarrow U_{i-1} .
\end{aligned}
$$

Consider now the formal series in $I_{i}(i=1, \ldots, 2 N), \ldots, \stackrel{(j)}{U_{i}}\left(i=1, \ldots, r_{j}\right) \ldots$ which we call a vertex series. We define the degree of the monomial

$$
\prod_{i=1}^{2 N}\left(I_{i}\right)^{\alpha_{2}} \ldots \prod_{i=1}^{r_{j}}\left(\stackrel{(U)}{i}^{\beta}\right)^{\beta L} \ldots
$$

by the total number of legs

$$
d=\sum_{i=1}^{2 N} i \alpha_{i}+\ldots+\sum_{i=1}^{r_{j}} i \beta_{i}+\ldots
$$

To each $2 d$-th degree monomial, we assign the sum over the $(2 d-1)$ !! diagrams that we can build from the vertices of this monomial. For example

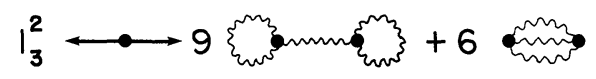

This correspondence is one-to-one, and if we extend it by linearity on the set of even vertex series, it becomes an isomorphism for the addition and the multiplication by a scalar.

If the monomial is odd, we cannot build a diagram from it. So, we assign zero to these odd monomials.

We want to study the problem of simplifying these Feynman diagram series. In Sect. IV, we briefly indicate an algebraic method to solve this problem, and in Sect. V, we apply it to the series $S_{I}$ (3.14). The principle of the method consists in replacing simplification rules on diagrams by algebraic operations on vertex series, owing to the previous isomorphism. These operations must preserve the parity of monomials. For a more comprehensive study of this algebraic treatment, the reader is referred to [13]. 


\section{B. Definitions}

(a) To begin with, we focus on the simplifications on diagrams and give some definitions which are inspired by those customarily used in graph theory.

(i) Partial graph: graph obtained by considering only the lines - (and neglecting --_-) and the vertices which are joined by them. It is generally made of several connected components.

(ii) Subgraph : graph obtained by considering a subset of vertices and all the lines which join them.

(iii) Tree: connected graph without cycles.

Let us now introduce the notions of simplifiable tree and root, which are basic to our study of simplifications.

Consider the graph $\mathrm{G}=$; denote by $G^{\prime}$ the partial graph of that one obtained by deleting the line $\left(A^{1}-B\right)$ and denote by $\Gamma$ the connected component of $G^{\prime}$ containing the vertex $B$.

The leg (1) attached to the vertex $A$ is said to be a root if and only if the partial subgraph $\left[\left(A^{1}-B\right) \cup \Gamma\right]$ is a tree whose vertices (except possibly the vertex $A$ ) are vertices $I_{i}(1 \leqq i \leqq 2 N)$.

Then the partial subgraph $\left[\left(A^{1}-B\right) \cup \Gamma\right]$ is called the simplifiable tree generated by the root (1).

A leg of a vertex is said to be simplifiable if and only if it satisfies one of the following conditions:

(i) it is a root of a simplifiable tree,

(ii) it belongs to a line

We must pay attention of the fact that this is nothing more than a definition. Indeed, a leg may be eliminated by simplifications without being simplifiable. On the other hand, it is easily seen that repeating the rules (2) and (3) consists in eliminating respectively simplifiable trees and lines

(b) The general idea of this study is to disconnect simplifiable legs from other legs by adding new vertices if necessary. If we examine closely the behaviour of vertices during simplifications, we are led to introduce four new types of vertex $(\tilde{I}, L, \tilde{U}, R)$. (2.16a).

To each vertex $I_{i}(i=1, \ldots, 2 N)$, we assign two vertices $\tilde{I}_{i}$ and $L_{i}$ defined as $I_{i}$ by

To each vertex $U_{i}(i=1, \ldots, r)$ of each family, we assign one vertex $\tilde{U}_{i}$ defined as $U_{i}$ by (4.1).

Moreover we define the vertex $R$ as follows

$$
R=\frac{\langle\mathbf{f} \mid \mathbf{Y}\rangle}{\left\langle\mathbf{f} \mid x_{0} \mathbf{u}\right\rangle}
$$

were $\mathbf{f}$ represents any path such that $\left\langle\mathbf{f} \mid x_{0} \mathbf{u}\right\rangle \neq 0$.

Finally, these vertices must satisfy the following conditions:

$\left(a_{1}\right)$ the $R^{\prime}$ leg is simplifiable;

$\left(\mathrm{a}_{2}\right)$ all the vertices $\tilde{I}_{i}$ and $\tilde{U}_{i}$ have no simplifiable leg;

$\left(\mathrm{a}_{3}\right) L_{i}(i=1, \ldots, 2 N)$ has at least $(i-1)$ simplifiable legs. 
More precisely, if we consider a monomial containing any type of vertex $(I, \tilde{I}, L, U, \tilde{U}, R)$ and if we apply the decomposition rule (1) on its associated Feynman diagram sum, we only keep diagrams whose vertices satisfy the conditions $\left\{a_{i}\right\}$ and we drop the other diagrams.

In what follows, it is convenient to identify

$$
\begin{aligned}
L_{1} & =I_{1} \\
L_{0} & =I_{0} \\
\tilde{U}_{0} & =U_{0} \text { for each family. }
\end{aligned}
$$

The vertex $R$ has been normalized so that the associated scalar $R_{0}=1$. It is independent of the path $\mathbf{f}$ that is used to define it.

\section{Algebraic Process of Simplification}

We now give some algebraic results, the proofs of which will be found in [13].

From the properties of the vertex $R$ and the simplification rules (2), (3), we can write two "disconnection" rules (4), (5) and two new simplification rules (6), (7), which are represented in Fig. 2. As indicated by their name, the first two ones enable us to disconnect a simplifiable leg from the other legs. By means of these rules, we can calculate the algebraic expression of the vertices

$$
\left\{I_{i}, i=2, \ldots, 2 N\right\}, \ldots,\left\{U_{i}, i=1 \ldots r\right\}, \ldots
$$

in terms of the other ones introduced in Sect. (IV.B). One finds

$$
\begin{aligned}
I_{i} & =L_{i}+\sum_{j=2}^{i} C_{i}^{j} \tilde{I}_{j} R^{i-j} \quad i \geqq 2 \\
U_{i} & =\sum_{j=0}^{i} C_{i}^{j} \tilde{U}_{j} R^{i-j}
\end{aligned}
$$

As for the simplification rules (2), (3), (6), (7), they are used to prove that the action of the vertices $R$ and $I_{1}$ on a vertex series $f$ is equivalent to derivation operators $D^{\prime}$ and $D^{\prime \prime}$ (with the usual rules for addition and product) characterized by its images on the basic vertices

with

$$
R f=D^{\prime}(f) \quad I_{1} f=D^{\prime \prime}(f)
$$

$$
\begin{aligned}
& D^{\prime}(R)=\frac{N}{2(N-1) I_{0}}, \\
& D^{\prime}\left(L_{i}\right)=i\left(-\frac{1}{2(N-1)}\right) R^{i-1}+i\left(\frac{N}{2(N-1) I_{0}}\right) L_{i-1}, \\
& D^{\prime}\left(\tilde{I}_{i}\right)=D^{\prime}\left(\tilde{U}_{i}\right)=0
\end{aligned}
$$

and

$$
\begin{aligned}
& D^{\prime \prime}(R)=\frac{1}{2} \\
& D^{\prime \prime}\left(L_{i}\right)=i\left(\frac{1}{2}\right) L_{i-1}, \\
& D^{\prime \prime}\left(\tilde{I}_{i}\right)=D^{\prime \prime}\left(\tilde{U}_{i}\right)=0 .
\end{aligned}
$$




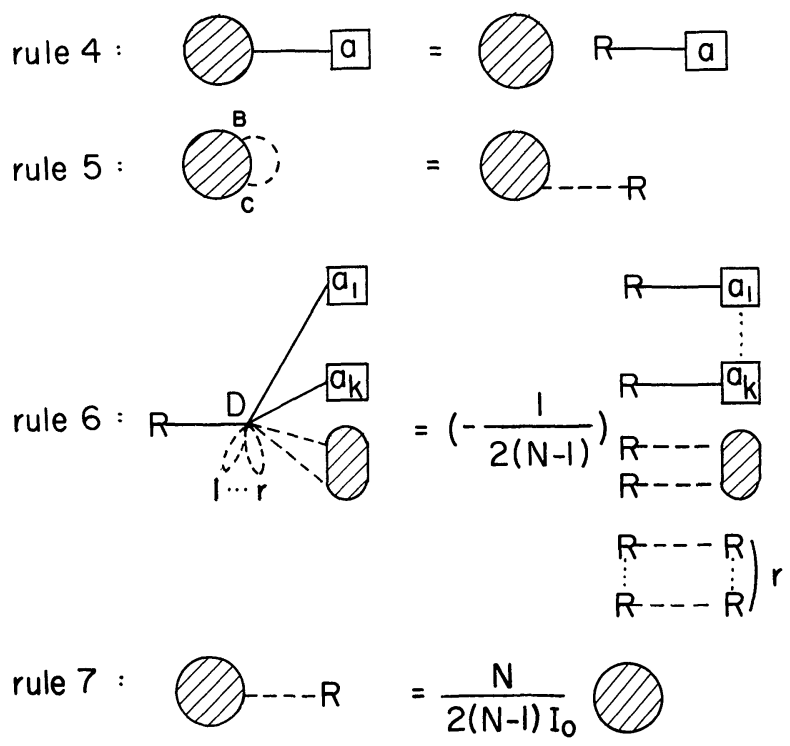

Fig. 2. Disconnection rules (4), (5) and simplification rules (6), (7). (i) $\bigotimes$ is any subgraph and $s$ is a simplifiable tree with root $s$. To keep the figure clear, the lines - - - between $\bigotimes, a, \ldots, a_{k}$ are not represented. (ii) In rule (5) $B$ and $C$ may represent the same vertex (loop). (iii) In rule (6), $D$ is any vertex $U_{i}$ or $L_{i}(i \geqq 1)$

Note that the actions of $D^{\prime}$ and $D^{\prime \prime}$ on the vertices of type $\{I\}$ or $\{U\}$ may be obtained merely by applying these derivations on Eqs. (4.7).

We still need another relation which we call "constant term relation"

$$
L_{i} f(L, \tilde{I}, \tilde{U})=I_{0} R^{i} f(L, \tilde{I}, \tilde{U}) .
$$

Equation (4.11) is valid for any series not containing $R$ (which also excludes vertices of type $\{U\}$ or $\left\{I_{i} i \geqq 2\right\}$ ).

We are now in a position to describe the algebraic process to simplify any vertex series $f(I, \tilde{I}, L, U, \tilde{U}, R)$ :

(i) By means of Eqs. (4.7), express the series in a sum of monomials of the form

$$
M=M_{1} M_{2}
$$

with

$$
\begin{aligned}
& M_{1}=R^{\alpha} \prod_{i=1}^{2 N} L_{i}^{\beta_{i}} \\
& M_{2}=\left(\prod_{i=1}^{2 N} \tilde{I}_{i}^{\gamma_{i}}\right) \ldots\left(\prod_{i=1}^{r} \tilde{U}_{i}^{\delta_{i}}\right) \ldots
\end{aligned}
$$

The simplification rules are concerned uniquely with $M_{1}$ whereas $M_{2}$ remains invariant during simplifications.

(ii) If $M_{1}$ is even, eliminated the vertices $R$ and $I_{1}$ by repeated application of the derivations $D^{\prime}$ and $D^{\prime \prime}$. When there is no more $R$ or $I_{1}$, proceed by using Eq. (4.11) and apply again the derivation $D^{\prime}$. And so on.... Whenever we use the 
derivations $D^{\prime}$ or $D^{\prime \prime}$, the degree of the monomial decreases by two units. The process stops when $M_{1}$ has degree zero, i.e. is reduced to a numerical coefficient.

(iii) If $M_{1}$ is odd, the contribution of $M$ with regard to Feynman diagrams is zero and we replace $M$ by 0 .

In this way, we can algebraically reduce any vertex series. Moreover, we deduce the following consequence: After simplification, any vertex series $f(I, \tilde{I}, L, U, \tilde{U}, R)$ may be expressed in terms of the only vertices of type $\tilde{I}$ and $\tilde{U}$.

To end this section, before simplifying $S_{I}(3.14)$, we have to define the algebraic framework and rules to solve this kind of problem.

\section{Formalism}

(a) Let us first give some definitions: (i) We denote by $\mathscr{A}\left[\frac{1}{\sqrt{K}}\right]$ the set of formal series with the indeterminate $1 / \sqrt{K}$
and real coefficients.

(ii) We denote by $\mathscr{S}$ the set of formal series with the indeterminate

$$
\left\{R, L_{i}(1 \leqq i \leqq 2 N), \tilde{I}_{i}(2 \leqq i \leqq 2 N), \ldots, \tilde{U}_{i}(1 \leqq i \leqq r), \ldots\right\}
$$

and whose coefficients belong to $\mathscr{A}[1 / \sqrt{K}]$. In addition, we define the indeterminates

$$
\left\{I_{i}(1 \leqq i \leqq 2 N), \ldots, U_{i}(1 \leqq i \leqq r), \ldots\right\}
$$

in terms of the previous ones by Eqs. (4.5), (4.7). $\mathscr{S}$ forms a commutative $\mathscr{A}$-algebra under addition, multiplication by an element of $\mathscr{A}\left[\frac{1}{\sqrt{K}}\right]$ and product.

(iii) We denote by $\mathscr{F}$ the mapping which assigns to a series $f \in \mathscr{S}$ its image $\mathscr{F}(f) \in \mathscr{S}$ after all the simplifications have been made

$$
f(R, L, \tilde{I}, \tilde{U}) \stackrel{\mathscr{F}}{\longrightarrow}(\mathscr{F} f)(\tilde{I}, \tilde{U}) .
$$

This mapping is well defined by the algebraic process [Sect. IV.C)] and it is a $\mathscr{A}$-linear operator.

(iv) We denote by $\mathbf{C T}(f)$ the coefficient of degree 0 of the image series $\mathscr{F}(f)$ and we call it "constant term of $f$ ". $\mathbf{C T}(f)$ represents the sum over graphs of $f$ which are entirely reduced by simplifications. For a monomial $M=M_{1} M_{2}(4.12)$, we have

$$
\mathscr{F}\left(M_{1} M_{2}\right)=M_{2} \mathbf{C T}\left(M_{1}\right)
$$

with, if $M_{1}$ is odd, $\mathbf{C T}\left(M_{1}\right)=0$.

For a series $f\left(I_{i}, \ldots, U_{i}, \ldots, R\right)$, it follows that

$$
\mathbf{C} \mathbf{T}\left(f\left(I_{i}, \ldots, U_{i}, \ldots, R\right)\right)=\mathbf{C} \mathbf{T}\left(f\left(L_{i}, \ldots, U_{0} R^{i}, \ldots, R\right)\right) .
$$

(v) Two series $f$ and $g$ of $\mathscr{S}$ are said to be equivalent when their images are identical

$$
f \sim g \Leftrightarrow \mathscr{F}(f)=\mathscr{F}(g) .
$$

This relation is an equivalence relation compatible with the addition and multiplication by an element of $\mathscr{A}\left[\frac{1}{\sqrt{K}}\right]$, but not with the product. In the following, the 
equivalence of two series is always referred to this definition. We have of course

$$
f \sim g \Rightarrow \mathbf{C T}(f)=\mathbf{C T}(g) .
$$

(b) Since $\mathscr{S}$ is a commutative $\mathscr{A}$-algebra, we may define derivations on it which are completely defined by the derivatives of the basic vertices.

(i) We define in $\mathscr{S}$ the derivations $\frac{\partial}{\partial \tilde{I}_{j}}(2 \leqq j \leqq 2 N) \ldots \frac{\partial}{\partial \widetilde{U}_{j}}(1 \leqq j \leqq r) \ldots$ which are the usual partial derivatives.

For a series $f\left(I_{i}, \ldots, U_{i}, \ldots, R\right)$, it follows from Eqs. (4.7) that

$$
\frac{\partial}{\partial \tilde{I}_{j}}(f)=\sum_{i=1}^{2 N} \frac{\partial I_{i}}{\partial \tilde{I}_{j}} \frac{\partial}{\partial I_{i}}(f)=\sum_{i \geqq j} C_{i}^{j} R^{i-j} \frac{\partial}{\partial I_{i}}(f)
$$

and similarly

$$
\frac{\partial}{\partial \tilde{U}_{j}}(f)=\sum_{i \geqq j} C_{i}^{j} R^{i-j} \frac{\partial}{\partial U_{i}}(f) .
$$

(ii) Consider now the monomial $M=M_{1} M_{2}$ (4.12). We have:

$$
\mathscr{F}\left(\frac{\partial}{\partial \tilde{I}_{j}}\left(M_{1} M_{2}\right)\right)=\mathbf{C T}\left(M_{1}\right) \frac{\partial}{\partial \tilde{I}_{j}}\left(M_{2}\right)=\frac{\partial}{\partial \tilde{I}_{j}}\left(\mathscr{F}\left(M_{1} M_{2}\right)\right)
$$

and an analogous result for $\frac{\partial}{\partial \tilde{U}_{j}}$. This is extended by linearity to any series of $\mathscr{S}$ :

$$
\left[\mathscr{F}, \frac{\partial}{\partial \tilde{I}_{j}}\right]_{-}=\left[\mathscr{F}, \frac{\partial}{\partial \tilde{U}_{j}}\right]_{-}=0 .
$$

(iii) We have seen in Sect. IV.C) that the operators $D^{\prime}$ and $D^{\prime \prime}$ were derivations characterized by (4.9), (4.10). They satisfy Eqs. (4.8) which must be now rewritten as:

$$
R f \sim D^{\prime}(f), \quad I_{1} f \sim D^{\prime \prime}(f) .
$$

The operator

$$
D=D^{\prime}-\frac{N}{(N-1) I_{0}} D^{\prime \prime}
$$

is also a derivation of $\mathscr{S}$ characterized by

$$
\begin{aligned}
& D\left(L_{i}\right)=i\left(-\frac{1}{2(N-1)}\right) R^{i-1}, \\
& D(R)=D\left(\tilde{I}_{i}\right)=D\left(\tilde{U}_{i}\right)=0 .
\end{aligned}
$$

For a series $f\left(I_{i}, \ldots, U_{i}, \ldots, R\right)$, it follows that

$$
D(f)=-\frac{1}{2(N-1)} \sum_{i=1}^{2 N} C_{i}^{1} R^{i-1} \frac{\partial}{\partial I_{i}}(f) .
$$

We have now introduced all that we need to solve the problem of simplifying series like $S_{I}$. 


\section{Simplification of $S_{I}$}

To begin this section, we derive a fundamental relation valid for certain series (in particular for $S_{I}$ ) and we deduce an interesting property of these series. The fundamental relation will be very useful to simplify $S_{I}$.

\section{A. Fundamental Relation}

Let $S(K, p)$ be the vertex series

$$
S(K, p)=\exp \left[(K+p) \log \left(\sum_{i=0}^{2 N} C_{2 N}^{i} \frac{I_{i}}{k^{i} K^{i / 2} I_{0}}\right)-\frac{2 N \sqrt{K} I_{1}}{k I_{0}}-C_{2 N}^{2} \frac{I_{2}}{k^{2} I_{0}}+2 \frac{N^{2} I_{1}^{2}}{k^{2} I_{0}^{2}}\right],
$$

where $p$ is any real and $k=\sqrt{\frac{2 N}{I_{0}}}$.

Consider now the vertex series $f(R, \tilde{U}, \tilde{I}) S(K, p)$ where $f(R, \tilde{U}, \tilde{I})$ is any series containing no vertex $L_{i}(1 \leqq i \leqq 2 N)$, which also excludes vertices $I_{i}(1 \leqq i \leqq 2 N)$. From Eqs. (4.20) and (4.21), we have

$$
\left(\frac{R}{k \sqrt{K}}-\frac{N}{(N-1) I_{0}} \frac{I_{1}}{k \sqrt{K}}\right) f(R, \tilde{U}, \tilde{I}) S(K, p) \sim \frac{1}{k \sqrt{K}} D[f(R, \tilde{U}, \tilde{I}) S(K, p)]
$$

and, using Eq. (4.23) and $k^{2} I_{0}=2 N$

$$
\begin{aligned}
\sim- & \frac{1}{2(N-1)}\left[\frac{K+P}{K}\left(1+\frac{R}{k \sqrt{K}}\right)^{2 N-1} S(K, p-1) f(R, \tilde{U}, \tilde{I})\right. \\
& \left.-\left(1+\frac{2 N-1}{k \sqrt{K}} R-\frac{2 N}{k I_{0}} \frac{I_{1}}{\sqrt{K}}\right) S(K, p) f(R, \tilde{U}, \tilde{I})\right] .
\end{aligned}
$$

Since the equivalence relation is preserved under addition, the terms $I_{1} S(K, p) f(R, \tilde{U}, \tilde{I})$ cancel out. On the other hand, we can replace $f(R, \tilde{U}, \tilde{I})$ by $f(R, \tilde{U}, \tilde{I})\left(1+\frac{R}{k \sqrt{K}}\right)^{-1}$. Finally we obtain:

$$
\frac{K+P}{K}\left(1+\frac{R}{k \sqrt{K}}\right)^{2 N-2} S(K, p-1) f(R, \tilde{U}, \tilde{I}) \sim S(K, p) f(R, \tilde{U}, \tilde{I})
$$

This is the required fundamental relation, valid for any vertex series $f(R, \tilde{U}, \tilde{I})$. We now give an immediate application of (5.2): After simplification, the image of any series $f(R, \tilde{U}) S(K, p)$ contains no vertex $\tilde{I}_{2}$ which, in turn, is equivalent to

$$
\frac{\partial}{\partial \tilde{I}_{2}}[f(R, \tilde{U}) S(K, p)] \sim 0
$$

since $\left[\mathscr{F}, \frac{\partial}{\partial \tilde{I}_{2}}\right]_{-}=0$. Using the expression (5.1) of $S(K, p)$ together with (4.17) and (5.2), Eq. (5.3) is easily established. 


\section{B. Simplification of $S_{I}$}

We want to simplify the vertex series $S_{I}(3.14)$ and, more generally, $S_{I}(K, p)$ defined by

$$
S_{I}(K, p)=J\left(U, V, \ldots, W_{i} \ldots\right) S(K, p)
$$

with

(i) $J\left(U, V, \ldots, W_{i} \ldots\right)$ given by (3.14),

(ii) $S(K, p)$ given by $(5.1)$.

$S_{I}(K, p)$ is a Feynman diagram series satisfying the properties of Sect. IV.A. It involves $(n+2)$ families of type $\{U\}$ with $r=1$ everywhere. Furthermore

$$
U_{0}=1, \quad V_{0}=1, \quad\left(W_{i}\right)_{0}=0, \quad i=1, \ldots, n .
$$

Thus we can use the results of the previous section. After simplification, the series $\mathscr{F}\left(S_{I}(K, p)\right)$ may be written as follows:

$$
\begin{aligned}
\mathscr{F}\left(S_{I}(K, p)\right)= & \sum_{\alpha, \beta, \gamma_{3}, \ldots, \gamma_{2 N}} \prod_{m=3}^{2 N} \tilde{I}_{m} \tilde{U}^{\alpha} \\
& \cdot\left[f_{\alpha, \beta, \gamma_{3}, \ldots, \gamma_{2 N}}(n-1,1) \tilde{V}^{\beta}-f_{\alpha, \beta, \gamma_{3}, \ldots, \gamma_{2 N}}(n-2,0) \frac{\delta_{i j}-u_{i} u_{j}}{k^{2} K} \tilde{W}_{i} \tilde{W}_{j}\right]
\end{aligned}
$$

with

$$
=\begin{gathered}
f_{\alpha, \beta, \gamma_{3}, \ldots, \gamma_{2 N}}(a, b) \\
=\mathbf{C T}\left\{\prod_{m=3}^{2 N} \frac{1}{\gamma_{m} !}\left(\frac{\partial}{\partial \tilde{I}_{m}}\right)^{\gamma_{m}} \frac{1}{\alpha !}\left(\frac{\partial}{\partial \tilde{U}}\right)^{\alpha} \frac{1}{\beta !}\left(\frac{\partial}{\partial \tilde{V}}\right)^{\beta}\left(1+\frac{U}{k \sqrt{K}}\right)^{a}\left(1+\frac{V}{k \sqrt{K}}\right)^{b} S(K, p)\right\},
\end{gathered}
$$

$a$ and $b$ being arbitrary real numbers. Combining Eq. (4.17) with (5.2), we find

$$
\frac{\partial}{\partial \tilde{I}_{m}} S(K, p)=\frac{C_{2 N}^{m}}{2 N(k \sqrt{K})^{m-2}}\left(1+\frac{R}{k \sqrt{K}}\right)^{2-m} S(K, p)
$$

and, from Eqs. (4.18)

$$
\frac{\partial}{\partial \tilde{U}}\left(1+\frac{U}{k \sqrt{K}}\right)=\frac{\partial}{\partial \tilde{V}}\left(1+\frac{V}{k \sqrt{K}}\right)=\frac{1}{k \sqrt{K}} .
$$

Then, if we carry out the differentiations and replace the vertices $U$ and $V$ by $R$, owing to the property (4.14), we finally get

$$
\begin{aligned}
f_{\alpha, \beta, \gamma_{3}, \ldots, \gamma_{2 N}}(a, b)= & \prod_{m=3}^{2 N} \frac{1}{\gamma_{m} !}\left(\frac{C_{2 N}^{m}}{2 N}\right)^{\gamma_{m}} C_{a}^{\alpha} C_{b}^{\beta} \\
& \cdot\left(\frac{1}{k^{2} K}\right)^{\ell} \mathbf{C T}\left\{\left(1+\frac{R}{k \sqrt{K}}\right)^{a+b-2 \ell} S(K, p)\right\},
\end{aligned}
$$

where

(i) $C_{x}^{y}$ denotes the coefficient $\frac{\Gamma(x+1)}{\Gamma(y+1) \Gamma(x-y+1)}$,

(ii) $\ell=\sum_{m=3}^{2 N} \frac{m-2}{2} \gamma_{m}+\frac{\alpha+\beta}{2}$. 
Inserting (5.9) into (5.5) and shifting the index $\ell$ in the second term, we have

$$
\begin{aligned}
& \mathscr{F}\left(S_{I}(K, p)\right)=\sum_{\ell=0}^{\infty} \frac{1}{K^{\ell} k^{2 \ell}} \mathbf{C T}\left\{\left(1+\frac{R}{k \sqrt{K}}\right)^{n-2 \ell} S(K, p)\right\} \\
& \cdot\left[\sum_{\sum_{m=3}^{2 N} \frac{m-2}{2 \beta} \gamma_{m}+\frac{\alpha+\beta}{2}=\ell \text { or } 1} \prod_{m=3}^{2 N} \frac{1}{\gamma_{m} !}\left(\frac{C_{2 N}^{m}}{2 N} \tilde{I}_{m}\right)^{\gamma_{m}} C_{n-1}^{\alpha} \tilde{U}^{\alpha} \tilde{V}^{\beta}\right. \\
& \left.-\sum_{m=3}^{2 N} \sum_{m=\frac{m-2}{2} \gamma_{m}+\frac{\alpha}{2}+1=\ell} \prod_{m=3}^{2 N} \frac{1}{\gamma_{m} !}\left(\frac{C_{2 N}^{m}}{2 N} \tilde{I}_{m}\right)^{\gamma_{m}} C_{n-2}^{\alpha} \tilde{U}^{\alpha}\left(\delta_{i j}-u_{i} u_{j}\right) \tilde{W}_{i} \tilde{W}_{j}\right] \text {. }
\end{aligned}
$$

The vertices $\tilde{I}_{m}, \tilde{U}, \tilde{V}, \tilde{W}_{i}$ have respectively the same form than $I_{m}, U, V, \dot{W}_{i}$ but they must be linked with the propagator $G$ instead of $\mathscr{G}$. We recognize in the term between brackets the general term $b_{\ell}(T)$ of the series $B(\mu, T)$ defined in (2.18). Then

$$
\mathscr{F}\left(S_{I}(K, p)\right)=\sum_{\ell=0}^{\infty} \frac{1}{k^{2 \ell} K^{\ell}} \mathbf{C T}\left\{\left(1+\frac{R}{k \sqrt{K}}\right)^{n-2 \ell} S(K, p)\right\} b_{\ell}(T) .
$$

It now remains to work out these constant terms. The calculation is rather technical and it is postponed to Appendix B. The final result is

$$
\mathbf{C T}\left\{\left(1+\frac{R}{k \sqrt{K}}\right)^{a} S(K, p)\right\}=\frac{\Gamma(K+p+1) e^{N K} \Gamma[(K+p)(N-1)+a / 2]}{2 \pi K^{N(K+p)+a / 2}(N-1)^{(K+p)(N-1)+a / 2-1 / 2}}
$$

valid for any real $a$ and $p$. Inserting (5.12) into (5.11), we get

$$
\mathscr{F}\left(S_{I}(K, p)\right)=\frac{e^{N K} \Gamma(K+p+1)}{2 \pi K^{N(K+p)+n / 2}} \sum_{\ell=0}^{\infty} \frac{\Gamma[(K+p)(N-1)+n / 2-\ell]}{(N-1)^{(K+p)(N-1)+n / 2-1 / 2}}\left(\frac{N-1}{k^{2}}\right)^{\ell} b_{\ell}(T) .
$$

$\mathscr{F}\left(S_{I}(K, p)\right)$ represents the image of $S_{I}(K, p)$ after simplification. For $p=0$, we find the announced result (3.18).

Remark. We observe that the constant terms (5.12) do not depend on $I_{0}$, i.e. on the period $T$. To see the reason for this, it is convenient to renormalize the vertices $I_{l} \rightarrow I_{i} I_{0}$. Then the simplification rules (2), (3) read as in Fig. 3 so that a factor proportional to $1 / I_{0}$ arises at each step of simplification. Furthermore, in $S(K, p)$ the exponent of the factor $1 / k$ in front of each vertex is equal to the number of legs of this vertex. Then, at each step of simplification of the series, a factor arises which is proportional to $1 / k^{2} I_{0}$, i.e. independent of $I_{0}$. Consequently, these constant terms appear as pure combinatorial coefficients independent of $I_{0}$ and they are uniquely determined by the application of simplification rules to the series $S(K, p)$. They will have the same expression for any integral, functional or ordinary, if we apply the same simplification rules on the same vertex series.

Fig. 3

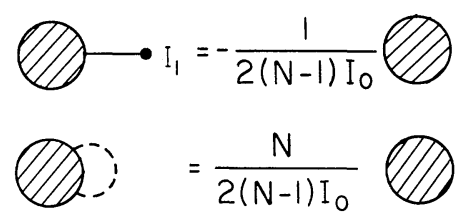




\section{Analytic Results for $N=2$}

We have seen that both methods give for $E_{K}$ the same asymptotic behaviour. For $N=2$, one finds

$$
E_{K} \sim-\frac{1}{\pi} \frac{6^{n / 2}}{\Gamma(n / 2)}(-3)^{K} \sum_{\ell=0}^{\infty} \Gamma\left(K+\frac{n}{2}-\ell\right)\left(\frac{4}{3}\right)^{\ell} a_{\ell}
$$

with $a_{0}=1$. The coefficient $a_{1}$ has been evaluated by Auberson et al. [8] and also Collins et al. [3] for $n=1$. In this section, we give $a_{1}$ for any $n, a_{2}$ for $n=1$, and we compare with the results of Bender and $\mathrm{Wu}$ [9] and Zinn-Justin [10].

\section{A. Preliminary}

For $n=1$, Auberson et al. [8] have proved that we can calculate the coefficients $a_{\ell}$ by using the $T \rightarrow \infty$ limit of the propagators $G_{L}\left(t, t^{\prime}\right), G_{0}\left(t, t^{\prime}\right)$ and also of the vertices. This property is also true for $G_{T}\left(t, t^{\prime}\right)$ and consequently for any $n$. Let us give these limits :

$$
\begin{gathered}
x_{0}(t) \rightarrow \frac{\sqrt{2}}{\cosh t}, \\
G_{0}\left(t, t^{\prime}\right) \rightarrow \frac{1}{2} \exp \left[-\left|t-t^{\prime}\right|\right], \\
G_{L}\left(t, t^{\prime}\right) \rightarrow \frac{\sinh t \sinh t^{\prime}}{4}\left[\frac{1}{\cosh ^{2} t}+\frac{1}{\cosh ^{2} t^{\prime}}-\frac{1}{\cosh ^{2} t \cosh ^{2} t^{\prime}}\right] \\
\pm \frac{1}{2}\left[u_{L}(t) v_{L}\left(t^{\prime}\right)-v_{L}(t) u_{L}\left(t^{\prime}\right)\right], \quad t \lessgtr t^{\prime}
\end{gathered}
$$

with

$$
\begin{aligned}
u_{L}(t) & \rightarrow \frac{\sinh t}{\cosh ^{2} t}, \\
v_{L}(t) \rightarrow- & \frac{\cosh t}{2}+\frac{3}{2 \cosh t}-\frac{3 t \sinh t}{2 \cosh ^{2} t}, \\
G_{T}\left(t, t^{\prime}\right) \rightarrow & \frac{1}{4}\left[\frac{\cosh t}{\cosh t^{\prime}}+\frac{\cosh t^{\prime}}{\cosh t}-\frac{1}{\cosh t \cosh t^{\prime}}\right] \\
\pm & \frac{1}{2}\left[u_{T}(t) v_{T}\left(t^{\prime}\right)-v_{T}(t) u_{T}\left(t^{\prime}\right)\right], \quad t \lessgtr t^{\prime}
\end{aligned}
$$

with

$$
\begin{gathered}
u_{T}(t) \rightarrow \frac{1}{\cosh t}, \\
v_{T}(t) \rightarrow-\frac{\sinh t}{2}-\frac{t}{2 \cosh t} .
\end{gathered}
$$

In the following, we represent the propagators as.

$$
\begin{aligned}
& G_{0}\left(t, t^{\prime}\right)=++++++ \\
& G_{L}\left(t, t^{\prime}\right)= \\
& G_{T}\left(t, t^{\prime}\right)=m m^{m}
\end{aligned}
$$


We also define

$$
y_{0}(t)=\frac{x_{0}(t)}{\sqrt{2}}=\frac{1}{\cosh t}
$$

and normalize the vertices as follows

Fig. 4

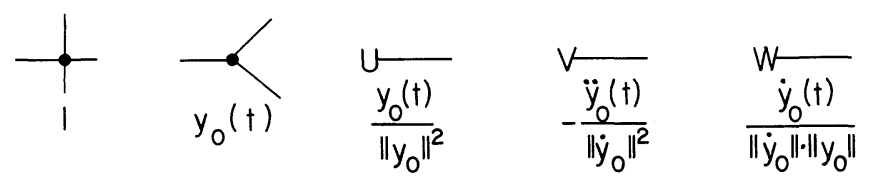

B. Coefficient $a_{1}$

For any $n, a_{1}$ is given by

$$
\begin{aligned}
a_{1}= & \frac{3}{4} a_{1}^{(\mathrm{a})}+3 a_{1}^{(\mathrm{b})}+9 a_{1}^{(\mathrm{c})}+6 a_{1}^{(\mathrm{d})} \\
& +(n-1)\left[\frac{1}{2} a_{1}^{(\mathrm{e})}+\frac{1}{2} a_{1}^{(\mathrm{f})}+6 a_{1}^{(\mathrm{g})}+2 a_{1}^{(\mathrm{i})}+a_{1}^{(\mathrm{j})}\right. \\
& \left.+3 a_{1}^{(\mathrm{k})}+\frac{1}{2} a_{1}^{(\mathrm{m})}-\frac{1}{4} a_{1}^{(\mathrm{n})}-\frac{1}{2} a_{1}^{(\mathrm{o})}\right] \\
& +(n-1)^{2}\left[\frac{1}{4} a_{1}^{(\mathrm{f})}+a_{1}^{(\mathrm{h})}+a_{1}^{(\mathrm{l})}+\frac{1}{4} a_{1}^{(\mathrm{n})}\right]
\end{aligned}
$$

where $a_{1}^{\text {(letter) }}$ represents the contribution of the corresponding diagrams of Fig. 5.

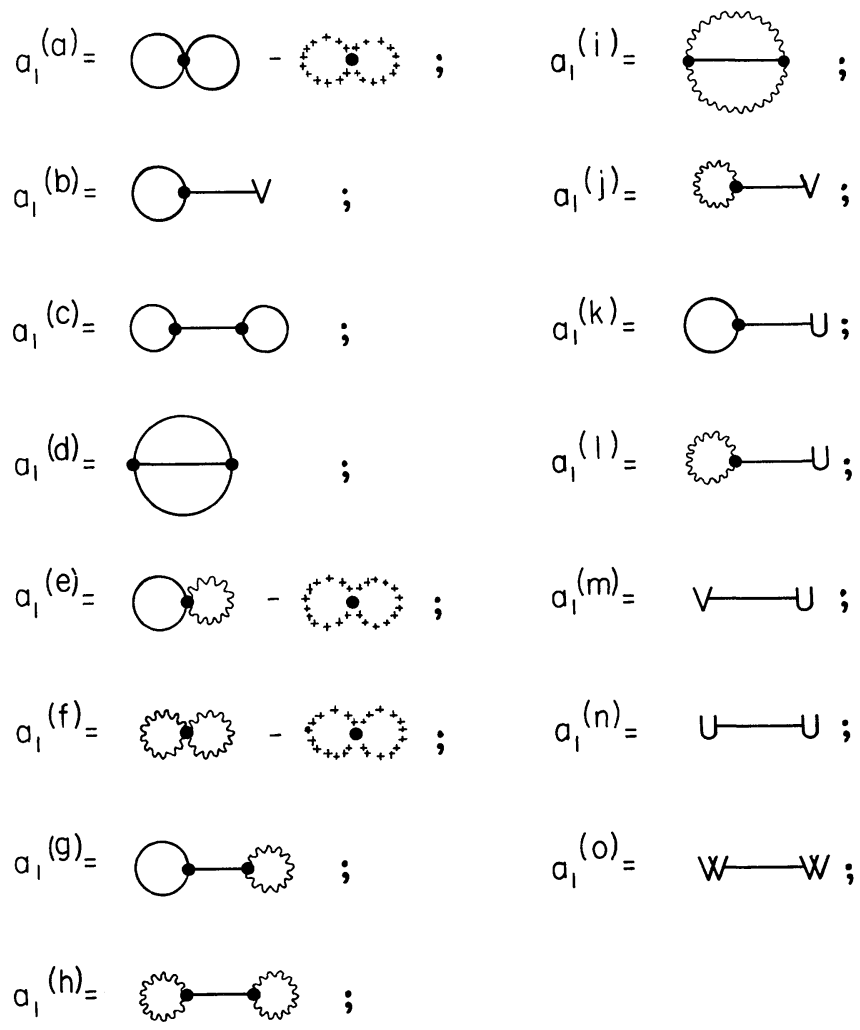

Fig. 5. The complete set of diagrams for the correction in $1 / K(N=2, n)$ 
Table 1. Numerical value of diagrams for the correction in $1 / K(N=2, n)$

\begin{tabular}{ll}
\hline$a_{1}^{(\mathrm{a})}=-\frac{319}{420} ;$ & $a_{1}^{(\mathrm{i})}=\frac{13}{960} ;$ \\
$a_{1}^{(\mathrm{b})}=-\frac{53}{240} ;$ & $a_{1}^{(\mathrm{j})}=-\frac{5}{16} ;$ \\
$a_{1}^{(\mathrm{c})}=\frac{13}{336} ;$ & $a_{1}^{(\mathrm{k})}=\frac{1}{144} ;$ \\
$a_{1}^{(\mathrm{d})}=-\frac{71}{4032} ;$ & $a_{1}^{(1)}=-\frac{1}{16} ;$ \\
$a_{1}^{(\mathrm{e})}=-\frac{13}{20} ;$ & $a_{1}^{(\mathrm{m})}=-\frac{3}{8} ;$ \\
$a_{1}^{(\mathrm{t})}=-\frac{5}{12} ;$ & $a_{1}^{(\mathrm{n})}=-\frac{1}{8} ;$ \\
$a_{1}^{(\mathrm{g})}=\frac{17}{720} ;$ & $a_{1}^{(\mathrm{o})}=\frac{3}{8} ;$ \\
$a_{1}^{(\mathrm{h})}=-\frac{1}{48} ;$ & \\
\hline
\end{tabular}

Their values are given in Table 1 and we obtain

$$
\frac{4}{3} a_{1}=-\frac{95}{72}-\frac{4}{3}(n-1)-\frac{7}{24}(n-1)^{2} .
$$

C. Coefficient $a_{2}$ (only for $n=1$ )

We have

$$
\text { Fig. } 6
$$

Figs. 6-8. The complete set of diagrams for the correction in $1 / K^{2}(N=2, n=1)$ 
Large Order Perturbation Expansion

425

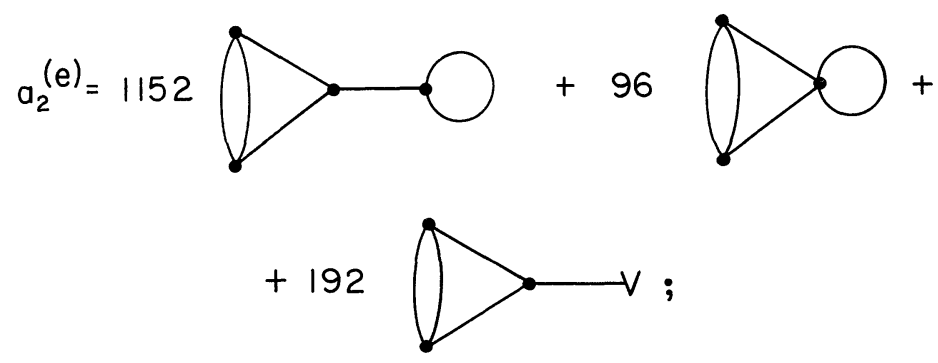

$$
a_{2}{ }^{(f)}=96
$$
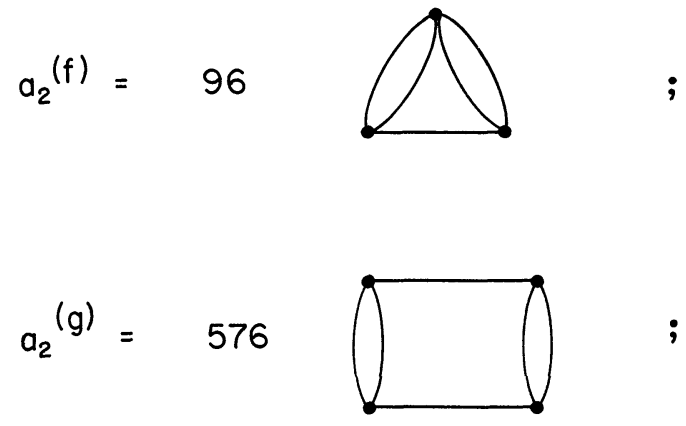

Fig. 7
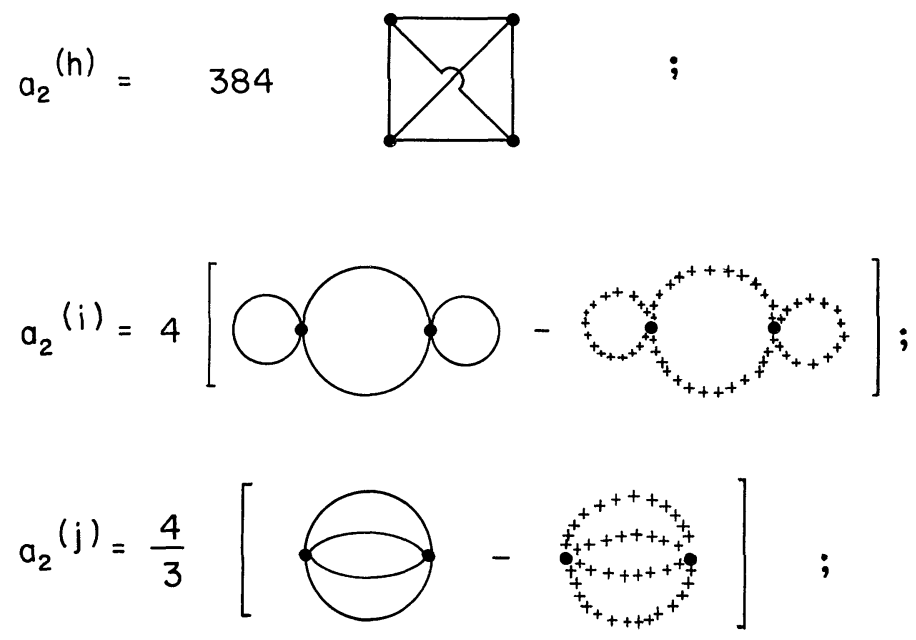

$$
\begin{aligned}
a_{2}^{(k)}= & \frac{1}{2 !}\left[a_{1}^{(a)}+12 a_{1}^{(c)}+8 a_{1}^{(d)}\right]^{2} \\
& +4 a_{1}^{(b)}\left[a_{1}^{(a)}+12 a_{1}^{(c)}+8 a_{1}^{(d)}\right] ;
\end{aligned}
$$

Fig. 8 
where $a_{2}^{\text {(letter) }}$ represents the contribution of the corresponding diagrams of Figs. 6 8. The exact (and quite tedious!) calculation of these quantities shows that we can express them as a linear combination of the four following integrals, the coefficients being always rational:

$$
\begin{aligned}
A & =\int_{-\infty}^{+\infty} \frac{t^{2}}{\cosh ^{2} t} d t=\frac{\pi^{2}}{6} \\
B & =\int_{-\infty}^{+\infty} \frac{t^{2}}{\cosh ^{2} t} \log (2 \cosh t) d t=\frac{\pi^{2}}{6}+\mathscr{Z}(3), \\
C & =\int_{-\infty}^{+\infty} \frac{t^{4}}{\cosh ^{2} t} d t=\frac{14}{15}\left(\frac{\pi}{2}\right)^{4}, \\
A^{2} & =\left[\int_{-\infty}^{+\infty} \frac{t^{2}}{\cosh ^{2} t} d t\right]^{2}=\frac{\pi^{4}}{36}
\end{aligned}
$$

$\mathscr{Z}$ represents the zeta function. The value of each contribution is given in Table 2 and we obtain

$$
\left(\frac{4}{3}\right)^{2} a_{2}=-\frac{13259}{2^{7} \cdot 3^{4}}
$$

Table 2. Numerical value of diagrams for the correction in $1 / K^{2}(N=2, n=1)$

$$
\begin{aligned}
& a_{2}^{(\mathrm{a})}=-\frac{7}{20} A-\frac{1439}{2^{5} \cdot 3^{4} \cdot 5 \cdot 7} ; \\
& a_{2}^{(\mathrm{b})}=-\frac{1763}{4900} A+\frac{723197}{2^{3} \cdot 3 \cdot 5^{3} \cdot 7^{3}} ; \\
& a_{2}^{\text {(c) }}=-\frac{17}{2450} A-\frac{300529}{2^{2} \cdot 3^{2} \cdot 5^{3} \cdot 7^{3}} ; \\
& a_{2}^{(\mathrm{d})}=\frac{2}{7} A-\frac{27}{140} B+\frac{4363}{2^{4} \cdot 3^{3} \cdot 5 \cdot 7} ; \\
& a_{2}^{(\mathrm{e})}=-\frac{221}{2450} A+\frac{27}{140} B-\frac{27076629}{2^{5} \cdot 3^{3} \cdot 5^{3} \cdot 7^{3}} ; \\
& a_{2}^{(\mathrm{f})}=\frac{2147}{4900} A-\frac{4311}{4900} B-\frac{27}{2450} C-\frac{81}{4900} A^{2}+\frac{6956653}{2^{4} \cdot 3^{3} \cdot 5^{2} \cdot 7^{3}} ; \\
& a_{2}^{(\mathrm{g})}=\frac{437}{490} A+\frac{1233}{4900} B+\frac{9}{4900} C+\frac{27}{9800} A^{2}-\frac{48932993}{2^{6} \cdot 3^{2} \cdot 5^{3} \cdot 7^{3}} ; \\
& a_{2}^{(\mathrm{k})}=\frac{124501}{2^{7} \cdot 5^{2} \cdot 3^{4}} \cdot \frac{2011}{2450} A+\frac{1233}{2450} B+\frac{9}{2450} C+\frac{27}{4900} A^{2}-\frac{37238309}{2^{6} \cdot 3^{4} \cdot 5^{3} \cdot 7^{3}} ; \\
& a_{2}^{(\mathrm{i})}=\frac{289}{4900} A-\frac{135403}{3 \cdot 5^{3} \cdot 7^{3}} ; \\
& a_{2}
\end{aligned}
$$


D. Asymptotic Behaviour of $E_{K}$ and Discussion

Inserting the values of $a_{1}$ and $a_{2}$ in (6.1), we obtain :

(i) for any $n$

$$
E_{K} \sim-\frac{1}{\pi} \frac{6^{n / 2}}{\Gamma(n / 2)}(-3)^{K} \Gamma\left(K+\frac{n}{2}\right)\left[1+\frac{1}{K}\left(-\frac{20+54 n+21 n^{2}}{72}\right)+O\left(\frac{1}{K^{2}}\right)\right],
$$

(ii) for $n=1$

$$
E_{K} \sim-\frac{1}{\pi} \sqrt{\frac{6}{\pi}}(-3)^{K} \Gamma\left(K+\frac{1}{2}\right)\left[1-\frac{95}{72 K}-\frac{20099}{10368 K^{2}}+O\left(\frac{1}{K^{3}}\right)\right] .
$$

This is equivalent to the results of Bender and $\mathrm{Wu}$ [9] and Zinn-Justin [10], which confirms the validity of functional approaches.

In the analytic calculation of $a_{2}$, we observe that the irrationals cancel out when we add all the diagrams. This fact had been conjectured by Bender and Wu [9] and it is reasonable to expect that the same is true at any order. However, the mechanism of this cancellation is still mysterious.

\section{Appendix A: A Theorem on Borel-Summable Functions}

Theorem. Let $f(z)$ be a Borel-summable function, such that

$$
\begin{aligned}
f(z) & =\sum f_{n} z^{n} \\
f_{n} & =c n ! a^{n} e^{i \varphi_{n}} n^{b}\left[1+O\left(\frac{1}{n}\right)\right]
\end{aligned}
$$

and $F(u)$ an analytic function in the circle $\left|u-f_{0}\right|<\varrho$.

Then

(i) the function

$$
g(z)=F(f(z))=\sum g_{n} z^{n}
$$

is Borel-summable,

(ii) for large $n, g_{n}$ is given at the order $\left(\frac{1}{n}\right)^{r} b y$

$$
g_{n}=\sum_{k=0}^{r} f_{n-k} d_{k},
$$

where $d_{k}$ is the generic term of the series

$$
\sum_{k} d_{k} \lambda^{k}=\left.\frac{d}{d u} F(u)\right|_{u=\sum_{k} f_{k} \lambda^{k}}
$$

Proof. (i) was shown by Auberson et al. [12].

(ii) is based on the following lemma. 
Lemma. Let $S$ be defined by

$$
S(n, p, r)=\sum_{\substack{\alpha_{1}+\ldots++\alpha_{p}=n \\ 1 \leqq \alpha_{i} \leqq n-r}} \alpha_{1} ! \ldots \alpha_{p} ! \alpha_{1}^{b} \ldots \alpha_{p}^{b}
$$

with $r \geqq p-1$. Then for $n$ large enough, $S$ is bounded by

$$
S(n, p, r) \leqq k(p, r)(n-r) !(n-r)^{b},
$$

where $k(p, r)$ is some constant. In particular

$$
k(p, p-1)=k^{p-1}, \quad k=2\left(1+2^{b}\right) .
$$

We shall prove the lemma by induction. It is true for $p=2$ :

$$
\begin{aligned}
S(n, 2, r) & =\sum_{q=r}^{n-r} q !(n-q) ! q^{b}(n-q)^{b} \\
& =2 r !(n-r) ! r^{b}(n-r)^{b}+\sum_{q=r+1}^{n-r-1} q !(n-q) ! q^{b}(n-q)^{b} .
\end{aligned}
$$

For $n$ large enough, all the terms of the latter sum are

$$
\leqq(r+1) !(n-r-1) !(r+1)^{b}(n-r-1)^{b} .
$$

Thus we have

$$
S(n, 2, r) \leqq 2 r !(n-r) ! r^{b}(n-r)^{b}\left[1+\frac{r+1}{2}\left(\frac{r+1}{r}\right)^{b}\right]
$$

namely

$$
S(n, 2, r) \leqq k(2, r)(n-r) !(n-r)^{b}
$$

with

$$
k(2,1)=2\left(1+2^{b}\right)
$$

For any $p, S(n, p, r)$ can be written

$$
S(n, p, r)=\sum_{q=r-(p-2)}^{n-r} q ! q^{b} S(n-q, p-1, p-2)+\sum_{q=1}^{r-(p-1)} q ! q^{b} S(n-q, p-1, r-q) .
$$

Using the induction hypothesis up to $p-1$, we get

$$
\begin{aligned}
S(n, p, r) & \leqq k^{p-2} S(n-p+2,2, r-p+2)+\sum_{q=1}^{r-(p-1)} q ! q^{b} k(p-1, r-q)(n-r) !(n-r)^{b} \\
& \leqq(n-r) !(n-r)^{b}\left[k^{p-2} k(2, r-p+2)+\sum_{q=1}^{r-(p-1)} q ! q^{b} k(p-1, r-q)\right]
\end{aligned}
$$

which is of the form (A.6) and proves the induction hypothesis.

Proof of (ii). We start from the general expression of $g_{n}$

$$
g_{n}=\sum_{p=1}^{n} F_{p} \sum_{\substack{\alpha_{1}+\ldots+\alpha_{p}=n \\ \alpha_{i} \geqq 1}} f_{\alpha_{1}} \ldots f_{\alpha_{p}}
$$


Let us separate this sum in three parts

$$
\begin{aligned}
g_{n}= & F_{1} f_{n}+\sum_{p=2}^{r+1} F_{p} \sum_{\substack{\alpha_{1}+\alpha_{p}=n \\
1 \leqq \alpha_{l} \\
\text { one of the } \alpha_{i} \geqq n-r}} f_{\alpha_{1}} \ldots f_{\alpha_{p}} \\
& +\sum_{p=2}^{r+1} F_{p} \sum_{\substack{\alpha_{1}+\ldots+\alpha_{p}=n \\
1 \leqq \alpha_{i} \leqq n-r-1}} f_{\alpha_{1}} \ldots f_{\alpha_{p}} \\
& +\sum_{p=r+2}^{n} F_{p} \sum_{\substack{\alpha_{1}+\ldots+\alpha_{p}=n \\
\alpha_{i} \geqq 1}} f_{\alpha_{1}} \ldots f_{\alpha_{p}} .
\end{aligned}
$$

From the behaviour of $f_{n}$, there exists $C(>c)$ such that

$$
\left|f_{\alpha}\right| \leqq C \alpha ! a^{\alpha} \alpha^{b}
$$

and, since $F(u)$ is analytical

$$
F_{p} \leqq \frac{K}{\varrho^{p}}
$$

Then we have

$$
(\mathrm{II})+(\mathrm{III}) \leqq \sum_{p=2}^{r+1} \frac{K}{\varrho^{p}} a^{n} C^{p} S(n, p, r+1)+\sum_{p=r+2}^{n} \frac{K}{\varrho^{p}} a^{n} C^{p} S(n, p, p-1) .
$$

Applying the previous lemma, we can write

$$
\begin{aligned}
(\mathrm{II})+(\mathrm{III}) \leqq & \left.K a^{n} \sum_{p=2}^{r+1}\left(\frac{C}{\varrho}\right)^{p} k(p, r+1)\right](n-r-1) !(n-r-1)^{b} \\
& +\frac{K a^{n}}{k}\left(\frac{k C}{\varrho}\right)^{r+2}(n-r-1) !(n-r-1)^{b} \\
& +\frac{K a^{n}}{k} \sum_{p=r+3}^{n}\left(\frac{k C}{\varrho}\right)^{p}[n-(p-1)] ![n-(p-1)]^{b} .
\end{aligned}
$$

For $n$ large enough, all the terms of this last sum are smaller than the first one. Thus, this sum will be majorized by

$$
\frac{K a^{n}}{k}\left(\frac{C k}{\varrho}\right)^{r+3}(n-r-1) !(n-r-1)^{b} .
$$

Hence

$$
(\mathrm{II})+(\mathrm{III}) \leqq C(r) a^{n}(n-r-1) !(n-r-1)^{b}
$$

and, from (A.8)

$$
(\mathrm{II})+(\mathrm{III}) \leqq C^{\prime}\left|f_{n}\right| \frac{1}{n^{r+1}} .
$$


Therefore, $g_{n}$ is given at the order $\left(\frac{1}{n}\right)^{r}$ by $(\mathrm{I})$, which is easily taken in the form

$$
g_{n}=F_{1} f_{n}+\sum_{k=1}^{r} f_{n-k} \sum_{\ell=2}^{k+1} \ell F_{\ell} \sum_{\substack{\alpha_{1}+\ldots+\alpha \ell-1 \\ 1 \leqq \alpha_{i} \leqq k-(\ell-2)}} f_{\alpha_{1}} \ldots f_{\alpha_{\ell-1}} .
$$

On the other hand, in the neighbourhood of $f_{0}$, we have

$$
\frac{d}{d u} F(u)=\sum_{\ell=1}^{\infty} \ell F_{\ell}\left(u-f_{0}\right)^{\ell-1}
$$

and, replacing $u-f_{0}$ by the series $\sum_{k=1}^{\infty} f_{k} \lambda^{k}$

$$
\left.\frac{d}{d u} F(u)\right|_{u=\sum_{k=0}^{\infty} f_{k} \lambda^{k}}=F_{1}+\sum_{k=1}^{\infty} \lambda^{k} \sum_{\ell=2}^{k+1} \ell F_{\ell} \sum_{\substack{\alpha_{1}+\ldots+\alpha_{\ell-1}=k \\ 1 \leqq \alpha_{i} \leqq k-(\ell-2)}} f_{\alpha_{1}} \ldots f_{\alpha_{\ell-1}} .
$$

By comparing (A.11) with (A.10), we obtain the theorem.

\section{Appendix B: Calculation of Constant Terms}

(a) These terms are even series of $\mathscr{A}\left[\frac{1}{\sqrt{K}}\right]$ with first coefficient equal to 1 :

$$
\mathbf{C T}\left[\left(1+\frac{R}{k \sqrt{K}}\right)^{a} S(K, p)\right]=1+\sum_{i=1}^{\infty} \frac{\alpha_{i}(p, a)}{K^{i}} .
$$

Since they depend on three variables, we need three recurrence relations to calculate them. The first one is merely the fundamental relation (5.2) applied to constant terms

$$
\frac{K+p}{K} \mathbf{C T}\left[\left(1+\frac{R}{k \sqrt{K}}\right)^{a+2 N-2} S(K, p-1)\right]=\mathbf{C T}\left[\left(1+\frac{R}{k \sqrt{K}}\right)^{a} S(K, p)\right] .
$$

(b) The second one is based on the "constant term relation" (4.11). To establish it, we start from the equation

$$
S(K, p)=\left(\sum_{i=0}^{2 N} C_{2 N}^{i} \frac{I_{i}}{k^{i} K^{i / 2} I_{0}}\right) S(K, p-1) .
$$

From (4.14) and (4.11), it follows that

$$
\mathbf{C T}[S(K, p)]=\mathbf{C T}\left[\left(1+\frac{R}{k \sqrt{K}}\right)^{2 N} S(K, p-1)\right] .
$$

In view of (B.2), (B.4) becomes

$$
\mathbf{C T}\left[\left(1+\frac{R}{k \sqrt{K}}\right)^{2} S(K, p)\right]=\frac{K+p}{K} \mathbf{C T}[S(K, p)] .
$$


We want to generalize Eq. (B.5) and prove that

$$
\mathbf{C T}\left[\left(1+\frac{R}{k \sqrt{K}}\right)^{a+2} S(K, p)\right]=\frac{K+p+\frac{a}{2(N-1)}}{K} \mathbf{C T}\left[\left(1+\frac{R}{k \sqrt{K}}\right)^{a} S(K, p)\right] \text { (B.6) }
$$

for any real $a$.

If we define the variable $F$ by

$$
1+F=\left(1+\frac{R}{k \sqrt{K}}\right)^{2 N-2}
$$

we have

$$
\left(1+\frac{R}{k \sqrt{K}}\right)^{a}=\sum_{\ell=0}^{\infty} \alpha_{\ell} F^{\ell}
$$

with

$$
\begin{aligned}
\frac{\alpha_{\ell+1}}{\alpha_{\ell}} & =\frac{\frac{a}{2 N-2}-\ell}{\ell+1} \\
\alpha_{0} & =1
\end{aligned}
$$

Let us introduce the quantities

$$
\begin{aligned}
& C(\ell, p)=\mathbf{C T}\left[F^{\ell} S(K, p)\right], \\
& D(\ell, p)=\mathbf{C T}\left[\left(1+\frac{R}{k \sqrt{K}}\right)^{2} F^{\ell} S(K, p)\right],
\end{aligned}
$$

with $\ell$ integer $\geqq 0$ and $p$ real. The dependence on the variable $K$ is implicit. From the fundamental relation (5.2), it follows that

$$
\begin{aligned}
& C(\ell, p+1)=\frac{K+p+1}{K}[C(\ell, p)+C(\ell+1, p)], \\
& D(\ell, p+1)=\frac{K+p+1}{K}[D(\ell, p)+D(\ell+1, p)] .
\end{aligned}
$$

We now prove by induction the following relation

$$
D(\ell, p)=\frac{K+p+\ell}{K} C(\ell, p)+\frac{\ell}{K} C(\ell-1, p) .
$$

This is true for $\ell=0$. Indeed, Eq. (B.5) may be rewritten

$$
D(0, p)=\frac{K+p}{K} C(0, p) .
$$


Assuming (B.13) to be true up to $\ell$, we have from (B.12)

$$
D(\ell+1, p)=\frac{K}{K+p+1} D(\ell, p+1)-D(\ell, p) .
$$

Using (B.13) for both terms in the right hand side, then (B.11), we get for Eq. (B.15) the expression (B.13) with $\ell \rightarrow \ell+1$. Relation (B.13) is then established.

Coming back now to our series, we have using (B.10), (B.13), and (B.9)

$$
\begin{aligned}
\mathbf{C T}\left[\left(1+\frac{R}{k \sqrt{K}}\right)^{a+2} S(K, p)\right] & =\sum_{\ell=0}^{\infty} \alpha_{\ell} D(\ell, p) \\
& =\sum_{\ell=0}^{\infty}\left(\alpha_{\ell} \frac{K+p+\ell}{K}+\alpha_{\ell+1} \frac{\ell+1}{K}\right) C(\ell, p) \\
& =\frac{K+p+\frac{a}{N-2} \sum_{\ell=0}^{\infty} \alpha_{\ell} C(\ell, p) .}{K}
\end{aligned}
$$

Relation (B.6) is then established by using (B.10a).

(c) Let $d(K, p, a)$ be the coefficient

$$
d(K, p, a)=\frac{2 K^{N(K+p)+a / 2}(N-1)^{(K+p)(N-1)+a / 2-1 / 2}}{\Gamma(K+p+1) e^{N K}} \frac{\mathbf{C T}\left[\left(1+\frac{R}{k \sqrt{K}}\right)^{a} S(K, p)\right]}{\Gamma\left[(K+p)(N-1)+\frac{a}{2}\right]} .
$$

If we replace the factors $\Gamma(K+p+1)$ and $\Gamma\left[(K+p)(N-1)+\frac{a}{2}\right]$ by the Stirling's asymptotic expansions and take account of (B.1), $d(K, p, a)$ is found to be an even series of $\mathscr{A}\left(\frac{1}{\sqrt{K}}\right)$ with first coefficient equal to $\frac{1}{\pi}$ :

$$
d(K, p, a)=\frac{1}{\pi}+\sum_{i=1}^{\infty} \frac{\beta_{i}(p, a)}{K^{i}} .
$$

Furthermore, the two recurrence relations (B.2) and (B.6) give respectively

$$
\begin{aligned}
d(K, p-1, a+2 N-2) & =d(K, p, a), \\
d(K, p, a+2) & =d(K, p, a) .
\end{aligned}
$$

We have seen that the constant terms are pure combinatorial coefficients which depend only on simplification rules and series $S(K, p)$. Consider now the path integral

$$
\begin{aligned}
\mathscr{I}= & \frac{1}{\Gamma(K+p+2)} \int d[X]\left(\int_{-T / 2}^{T / 2} X^{2}(t) d t\right)^{\frac{a-1}{2}}\left(\int_{-T / 2}^{T / 2} X^{2 N}(t) d t\right)^{K+p+1} \\
& \cdot \exp \left[-\int_{-T / 2}^{T / 2}\left(\frac{\dot{X}^{2}(t)}{2}+\frac{X^{2}(t)}{2}\right) d t\right]
\end{aligned}
$$


with $a, p$ reals and $n=1$. We may evaluate $\mathscr{I}$ by using the second method (Sect. III) in two ways according to how we insert in the exponential

$$
\left(\int_{-T / 2}^{T / 2} X^{2 N} d t\right)^{K} \text { or }\left(\int_{-T / 2}^{T / 2} X^{2 N} d t\right)^{K+1}
$$

By developing the calculations of Sect. III, applying the simplification procedure of Sect. IV and V and using the recurrence formula (B.6), we finally arrive at the equality

$$
d(K+1, p, a)=d(K, p+1, a) .
$$

Then Eqs. (B.18)-(B.20) together imply

$$
d(K+1, p, a)=d(K, p, a) .
$$

Combining (B.21) with (B.17), one gets

$$
d(K, p, a)=\frac{1}{\pi} .
$$

The required formula (5.12) is then established.

Acknowledgements. The author is grateful to Drs. G. Auberson and G. Mennessier for their encouragements, helpful suggestions and critical reading of this paper.

\section{References}

1. Bender, C.M., Wu, T.T.: Phys. Rev. 184, 1231-1260 (1969)

2. Lipatov, L.N.: Zh. Eksp. Teor. Fiz. 71, 2010-2024 (1976); 72, 411-427 (1977)

3. Collins, J.C., Soper, D.E.: Ann. Phys. 112, 209-234 (1978)

4. Seznek, R.: $3^{\mathrm{d}}$ Cycle Thesis, Saclay (1979)

5. Loeffel, J.J., Martin, A., Simon, B., Wightman, A.S.: Phys. Lett. 30B, 656-658 (1969)

6. Graffi, S., Grecchi, V., Simon, B.: Phys. Lett. 32B, 631-634 (1970)

7. Brézin, E., Le Guillou, J.C., Zinn-Justin, J.: Phys. Rev. D15, 1544-1564 (1977)

8. Auberson, G., Mahoux, G., Mennessier, G.: Nuovo Cimento 48A, 1-23 (1978)

9. Bender, C.M., Wu, T.T.: Phys. Rev. D7, 1620-1636 (1973)

10. Zinn-Justin, J.: Cen Saclay, preprint Dph-T-160 (1980)

11. For the method of collectives coordinates, see e.g., the footnote [7] of [7]. For the Faddeev-Popov method, see e.g., Fadde'ev, L.D., Popov, V.N.: Phys. Lett. 25B, 29-30 (1969); see also Jevicki, A., Nucl. Phys. B117, 365-376 (1976)

12. Auberson, G., Mennessier, G.: Montpellier Preprint PM/80/10

13. Figerou, V.R.: $3^{\mathrm{d}}$ Cycle Thesis, Montpellier (1980)

Communicated by J. Ginibre

Received August 1, 1980 
